\title{
The ectomycorrhizal basidiomycete Laccaria bicolor releases a GH28 polygalacturonase that plays a key role in symbiosis establishment
}

Feng Zhang ${ }^{1,2}$, Aurore Labourel ${ }^{3}$, Mireille Haon ${ }^{3}$, Minna Kemppainen ${ }^{4}$, Emilie Da Silva

5 Machado $^{2}$, Nicolas Brouilly ${ }^{5}$, Claire Veneault-Fourrey ${ }^{2}$, Annegret Kohler ${ }^{2}$, Marie-

Noëlle Rosso ${ }^{3}$, Alejandro Pardo ${ }^{4}$, Bernard Henrissat ${ }^{6,7,8}$, Jean-Guy Berrin ${ }^{3}$, Francis

$\operatorname{Martin}^{2,9}$

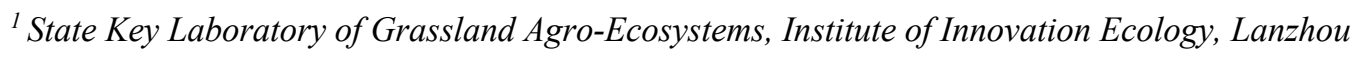

10 University, Lanzhou, 73000, China ${ }^{2}{ }^{2}$ Université de Lorraine, INRA, UMR 'Interactions

Arbres/Microorganismes', Laboratoire d'Excellence ARBRE, INRA-Grand Est, 54280, Champenoux,

France; ${ }^{3}$ INRA, Aix-Marseille Université, UMR 1163, Biodiversité et Biotechnologie Fongiques, 13009,

Marseille, France, ${ }^{4}$ Laboratorio de Micología Molecular, Instituto de Microbiología Básica y Aplicada,

Departamento de Ciencia y Tecnología, Universidad Nacional de Quilmes and Consejo Nacional de

15 Investigaciones Cientificas y Técnicas (CONICET), Bernal, Provincia de Buenos Aires, Argentina; ${ }^{5}$ AixMarseille Université, CNRS, IBDM, Marseille, France ; ${ }^{6}$ CNRS, UMR 7257 \& Architecture et Fonction des Macromolécules Biologiques, Aix-Marseille Université, Marseille, France; ${ }^{7}$ INRA, USC 1408 AFMB, 13288 Marseille, France; ${ }^{8}$ Department of Biological Sciences, King Abdulaziz University, Jeddah, Saudi Arabia $:{ }^{9}$ Beijing Advanced Innovation Center for Tree Breeding by Molecular Design, Beijing Forestry

\section{Authors for correspondence:}

Francis Martin (Email: francis.martin@inra.fr) and Feng Zhang (zf@lzu.edu.cn)

Received: ; Accepted:

Short title: A fungal pectinase plays a key role in the establishment of the ectomycorrhizal symbiosis 
Total: 5367 words: Summary: 180 / Introduction: 749 / Material and methods: 2409 / Results: 1086 / Discussion: 804 / Acknowledgements: 128

30 Figures: 8; Supplemental figures: 2.

\section{Summary}

- In ectomycorrhiza, root penetration and colonization of the intercellular space by symbiotic hyphae is thought to rely on the mechanical force that results from hyphal tip

35 growth, enhanced by the activity of secreted cell-wall-degrading enzymes.

- Here, we characterize the biochemical properties of the symbiosis-induced polygalacturonase LbGH28A from the ectomycorrhizal fungus Laccaria bicolor. The transcriptional regulation of $L b G H 28 A$ was measured by qPCR. The biological relevance of $L b G H 28 A$ was confirmed by generating RNAi-silenced $L b G H 28 A$

40 mutants. We localized the LbGH28A protein by immunofluorescence confocal and immunogold cytochemical microscopy in poplar ectomycorrhizal roots.

- $\quad$ qPCR confirmed the induced expression of $L b G H 28 A$ during ectomycorrhiza formation. L. bicolor RNAi mutants have a lower ability to establish ectomycorrhiza confirming the key role of this enzyme in symbiosis. The purified recombinant

45 LbGH28A has its highest activity towards pectin and polygalacturonic acid. In situ localization of $\mathrm{LbGH} 28 \mathrm{~A}$ indicates that this endopolygalacturonase is located in both fungal and plant cell walls at the symbiotic hyphal front.

- The present findings suggest that the symbiosis-induced pectinase LbGH28A is involved in the Hartig net formation and is an important determinant for successful symbiotic colonization.

Key words: apoplastic effector, carbohydrate-active enzymes, cell wall-modifying enzymes, pectins, polygalacturonase. 


\section{Introduction}

In boreal, mountainous and temperate forest ecosystems, trees rely on ectomycorrhizal associations to obtain nitrogen, phosphorus and micronutrients available in soils (Read et al., 2004; Smith \& Read, 2008; van der Heijden et al., 2015). Ectomycorrhizal fungi establish a symbiotic mutualistic interaction with host rootlets leading to morphogenetic

60 and metabolic changes in both fungal and plant symbionts (Brundrett, 2002; Peterson \& Massicotte, 2004). During root colonization, symbiotic hyphae first differentiate to form a pseudoparenchymatous mantle, a structure that encloses root apices (Massicotte et al., 1989). Subsequently, the hyphae grow between rhizodermal cells forming an intraradical, apoplastic hyphal network, so-called the Hartig net (Balestrini \& Kottke,

65 2016). The development of the Hartig net is initiated from the innermost layer of the mantle sheath and radial ingress of finger-like hyphae takes place in a broad lobed hyphal front (Balestrini \& Kottke, 2016). This labyrinthine hyphal structure is a key feature of the functional ectomycorrhiza. The fusion of fungal and plant cell wall polysaccharides and proteins in the Hartig net produces a common apoplast named the symbiotic interface. This is where the bi-directional translocation of metabolites takes place between the symbionts (Smith \& Read, 2008). Differentiation of the Hartig net is linked to subtle biochemical changes of both hyphae and cortical cell surfaces (Balestrini \& Kottke, 2016). Localized loosening and swelling, and redistribution of unesterified pectins, have been reported for plant cell walls at the symbiotic interface

75 (Balestrini et al., 1996; Balestrini \& Bonfante, 2014; Sillo et al. 2016). In addition, immunocytochemical microscopy has revealed changes in the spatial distribution of fungal cell wall proteins, such as hydrophobins and symbiosis-regulated acidic polypeptides, in the ectomycorrhizal Pisolithus microcarpus interacting with Eucalyptus globulus (Laurent et al., 1999; Tagu et al., 2001).

80 Colonization of the root middle lamella by ectomycorrhizal hyphae is thought to mainly rely on the mechanical force generated by the hydrostatic pressure at the tip of 
growing hyphae (Peterson \& Massicotte, 2004). It has also been proposed that auxins, released by colonizing hyphae, could promote root cell wall loosening (Gay et al., 1994ab), thus facilitating hyphal ingress. We have suggested that fungal plant cell walldegrading enzymes (PCWDEs) could also be involved in the middle lamella colonisation by symbiotic hyphae in L. bicolor-Populus ectomycorrhizal roots (Martin et al., 2008; Veneault-Fourrey et al., 2014). Similarly, Sillo et al. (2016) have shown by using the comprehensive microarray polymer profiling (CoMPP) technology that a localized degradation of pectin occurs during root colonization in Tuber

90 melanosporum-Coryllus avellana ectomycorrhizas. We recently reported that the symbiosis-induced B-1,4-endoglucanase LbGH5-CBM1 of the ectomycorrhizal fungus Laccaria bicolor is a secreted fungal endocellulase acting on poplar cell walls and this enzyme is an important determinant for successful symbiotic fungal colonization (Zhang et al., 2018). LbGH5-CBM1 expression is substantially induced in

95 ectomycorrhizal poplar roots and L. bicolor RNAi-silenced mutants with decreased LbGH5-CBMI expression have a lower ectomycorrhiza formation rate. In situ localization of LbGH5-CBM1 in ectomycorrhizal rootlets shows that this endoglucanase is located in cell walls of hyphae forming the Hartig net and mantle sheath. In addition to LbGH5-CBM1, three L. bicolor genes coding for pectinases

100 (polygalacturonases) of the glycoside hydrolase family 28 (GH28) are induced in L. bicolor-Populus ectomycorrhizal roots (Veneault-Fourrey et al., 2014), suggesting that the Hartig net development may require pectin degradation as shown in T. melanosporum-C. avellana ectomycorrhizas (Sillo et al., 2016).

Pectins are plant polysaccharides, mainly composed of D-galacturonic acid (GalA), 105 present in the middle lamella and in the primary and secondary cell walls, where they accumulate during the early stages of cell expansion. Pectins are classified, based on their chemical composition, in four primary types: homogalacturonan, rhamnogalacturonan I, xylogalacturonan and rhamnogalacturonan II. This linear 
polymer is formed of 100-200 GalA units linked by $\alpha-(1 \rightarrow 4)$ glycosidic bonds

110 (Christiaens et al. 2016). Polygalacturonases are hydrolytic enzymes that specifically cleave the $\alpha(1-4)$ bonds between adjacent galacturonic acids in pectin polygalacturonates.

In this study, we functionally characterized the ectomycorrhiza-induced endopolygalacturonase LbGH28A of L. bicolor. qRT-PCR assay showed that the

115 expression of $\mathrm{LbGH} 28 \mathrm{~A}$ gene is barely detectable in free-living mycelium, while it is strikingly upregulated in ectomycorrhizal root tips. The expression of $L b G H 28 A$ is induced by pectin and polygalacturonic acid suggesting a role in pectin metabolism. Biochemical characterization of the recombinant protein confirmed that LbGH28A is a pectinase. $L b G H 28 A$ RNAi-mutants show reduced mycorrhiza and Hartig net

120 formation, indicating an important role for this gene in the establishment of the symbiotic interface. By indirect immunofluorescence confocal microscopy, we also showed that LbGH28A accumulates at the periphery of hyphae in the Hartig net and mantle. Immunogold cytolocalization by transmission electron microscopy showed that LbGH28A is accumulating in the cell walls at the symbiotic interface. Our findings suggest that the symbiosis-induced LbGH28A pectinase plays a key role in the establishment of ectomycorrhizal symbiosis in concert with other symbiosis-induced PCWDEs.

\section{Material and Methods}

130 Biological material and growth conditions

Laccaria bicolor (Maire) P.D. Orton, dikaryotic strain S238N, was grown on a lowglucose Pachlewski agar medium (P20) (Pachlewski, 1967), on cellophane membranes placed on the medium surface, at $20^{\circ} \mathrm{C}$ in the dark. The axenic cultures were subcultured for mycorrhiza establishment weekly (Felten et al., 2009). Briefly, P20 is a 135 modification of Pachlewski's medium composed of $0.25 \mathrm{~g} \mathrm{~L}^{-1}$ di-ammonium tartrate, 
$0.5 \mathrm{~g} \mathrm{~L}^{-1} \mathrm{KH}_{2} \mathrm{PO}_{4}, 0.25 \mathrm{~g} \mathrm{~L}^{-1} \mathrm{MgSO}_{4}, 1.0 \mathrm{~g} \mathrm{~L}^{-1}$ glucose, $1 \mathrm{~mL} \mathrm{~L}^{-1}$ (diluted 1/10)

Kanieltra microelement solution and $20 \mathrm{~g} \mathrm{~L}^{-}$agar ${ }^{1}$. To assess the effect of galacturonic acid and pectin on $L b G H 28 A$ expression, L. bicolor $\mathrm{S} 238 \mathrm{~N}$ mycelium was cultured in $100 \mathrm{~mL}$ modified Melin Norkrans (MMN) medium for three days before being

140 transferred to a fresh MMN medium containing either $0.5 \% \mathrm{w} / \mathrm{v}$ glucose, polygalacturonic acid (P3889, Sigma-Aldrich, France) or pectin from Citrus peel (polyD-galacturonic acid methyl ester (galacturonic acid $\geq 74.0 \%$ dried basis)), P9135, Sigma-Aldrich), as the sole carbon source. Cultures were maintained in $250 \mathrm{~mL}$ flasks at $20^{\circ} \mathrm{C}$ in the dark on a rotary shaker $(200 \mathrm{rpm})$ for one week. Then, the mycelium was

145 harvested by filtration through a Büchner funnel under vacuum, washed twice with Milli-Q water, snap frozen in liquid $\mathrm{N}_{2}$ and stored at $-80^{\circ} \mathrm{C}$ for further RNA extraction (Dietz et al., 2011).

Hybrid grey poplar (Populus x canescens, synonym: Populus tremula $\mathrm{x}$ alba, clone INRA 717-1-B4) was used for in vitro ectomycorrhizal inoculation. Two-cm-long

150 cuttings were rooted on solid Murashige and Skoog (MS) medium (Felten et al., 2009), for three weeks, and mycelium of L. bicolor S238N (wild-type or RNAi-silenced lines) were grown on cellophane membranes on low-glucose Pachlewski agar medium in the dark for 10 days. Membranes with L. bicolor mycelium and poplar seedlings with one or two main roots were transferred onto the surface of low-glucose Pachlewski agar

155 medium $(0.1 \%$ glucose) containing $0.1 \%$ MES (M8250, Sigma, France), and covered by another cellophane membrane (i.e., "sandwich technique") (Felten et al., 2009). Inoculated and control plantlets were grown in a controlled environment growth chamber for $16 \mathrm{~h}\left(22^{\circ} \mathrm{C} / 18^{\circ} \mathrm{C}\right.$, day/night $)$ at $50-60 \%$ relative humidity and $400 \mu \mathrm{mol}$ $\mathrm{m}^{-2} \mathrm{~s}^{-1}$ photosynthetic photon flux density. Ectomycorrhizal rootlets, non-mycorrhizal 160 roots and extramatrical hyphæ were sampled three weeks after contact, snap frozen in liquid $\mathrm{N}_{2}$ and stored at $-80^{\circ} \mathrm{C}$ until further analyses. The rate of ectomycorrhiza formation was assessed as described in Felten et al. (2009). 


\section{Analysis of the $L b G H 28 A$ gene}

165 The gene coding for the predicted polygalacturonase (pectinase) LbGH28A of the glycoside hydrolase (GH) 28 family was identified by automatic and manual annotations in the genome of L. bicolor $\mathrm{S} 238 \mathrm{~N}-\mathrm{H} 82$ (http://genome.jgi.doe.gov/Lacbi2/Lacbi2.home.html; Joint Genome Institute ID \#313960 in v1 and JGI ID \#613299 in v2, GenBank accession \# XP_001877488.1). The

170 coding sequence of $L b G H 28 A$ was corrected based on Sanger sequencing of its cDNA. Approximately 50 ng of fungal total RNA, extracted using the Qiagen RNAeasy kit (Qiagen, Courtaboeuf, France) from ectomycorrhizal rootlets, was used for the first strand cDNA synthesis following the manufacturer's instructions (SMARTer RACE cDNA Amplification Kit (Clontech, Cat. No.634923)). The degenerated oligo(dT) and

175 3'-RACE and 5'-RACE primers used are listed in Supporting Information Table S1. Then, the amplified fragment was cloned in pJET1.2 vector (Thermo Scientific, K1231) and sequenced by Sanger sequencing. The full-length $L b G H 28 A$ cDNA was used as the reference transcript for producing the recombinant protein in yeast.

The nucleotide sequence of the $L b G H 28 A$ gene and its coding sequence was

180 analyzed and compared to sequences deposited in international databases (MycoCosm, GenBank, SwissProt) by using available online tools (https://mycocosm.jgi.doe.gov/mycocosm/home; http://www.ncbi.nlm.nih.gov/, http://www.expasy.org). The putative signal peptide of the LbGH28A protein sequence was predicted using the SignalP 4.1 server (http://www.cbs.dtu.dk/services/SignalP/).

185 The phylogenetic neighbor-joining tree was deduced from the alignment of fungal GH28 proteins using MAFFT (version 7) with the consistency based method and built the tree by using FastTree (version 2.1.11) and MEGAX (version 10.1.8). 


\section{Quantitative RT-PCR}

190 Axenic mycelium of L. bicolor S238N (100 mg fresh weight) and 25 to 50 mycorrhizal or non-mycorrhizal lateral rootlets (100 mg fresh weight), sampled on five different root systems of $P$. tremula $\mathrm{x}$ alba colonized by L. bicolor, were ground in liquid nitrogen using a mortar and pestle. Total RNA was extracted using the RNeasy Plant kit (Qiagen) according to the manufacturer's instructions with the addition of polyethylene

195 glycol 8000 to RLC buffer $\left(25 \mathrm{mg} \mathrm{mL}^{-1}\right)$. To avoid any DNA contamination, a DNA digestion step was performed on-column with DNAse I (Qiagen). RNA quality was checked by using Experion HighSens capillary gels (BioRad, Marnes-la-Coquette, France). Synthesis of cDNA from one $\mu \mathrm{g}$ of total RNA was performed using the iScript kit (\#1708891, BioRad, France) according to the manufacturer's instructions. All

200 primers were ordered from Eurogentec (Angers, France) and PCR amplification was performed using Taq DNA Polymerase (Thermo Fisher Scientific, France) according to the manufacturer's instructions and optimized according to each primer pairing. Realtime PCR analyses were performed using the Fast SYBR Green Master Mix (Applied Biosystems, France) with a final concentration of $0.3 \mu \mathrm{M}$ of each primer following the manufacturer's instructions. The thermal-cycling condition parameters of the StepOnePlus System qPCR apparatus (Applied Biosystems, France) were as follows: $95^{\circ} \mathrm{C}$ for $3 \mathrm{~min} ; 40$ cycles of $95^{\circ} \mathrm{C}$ for $15 \mathrm{~s}, 60^{\circ} \mathrm{C}$ for $30 \mathrm{~s}$ followed by a melting curve. PCR amplifications were carried out on three biological replicates and included two distinct technical replicates. Transcript abundance was normalized using four

210 constitutively expressed L. bicolor genes coding for a histone H4 (JGI ID\# 319764), ubiquitin (JGI ID\# 446085), a heat shock protein HSP70 (JGI ID\# 609242) and a mitochondrial substrate carrier protein (JGI ID\# 611151). Primer sequences (Supporting Information Table S1) were designed using the nucleotide sequences retrieved from the L. bicolor genome v2.0 (http://genome.jgi.doe.gov/Lacbi2/Lacbi2.home.html) and 215 available online tools (https://www.ncbi.nlm.nih.gov/tools/primer-blast/). Transcript 
abundance was quantified using the standard curve method of quantification (based on $\Delta \Delta \mathrm{Ct}$ calculations), as previously described by Pfaffl (2001).

\section{Generation of transgenic $L$. bicolor strains}

220 LbGH28A RNAi was performed using the RNAi/Agrobacterium-mediated transformation vector for intron hairpin RNA (ihpRNA) expression, and transformation of $L$. bicolor vegetative mycelium used the $\mathrm{pHg} / \mathrm{pSILBA} \gamma$ vector system as described in Kemppainen and Pardo (2010) using cDNA fragments of LbGH28A. A 495 bpsequence (positions 102 to 596) from the coding sequence was targeted for hairpin

225 formation in avoiding the homologous domain shared by other $\mathrm{LbGH} 28$ genes (Supplemental Information Fig. S2). Four randomly selected strains of each $L b G H 28 A$ RNAi-silenced and mock ( $\mathrm{pHg} /$ empty pSILBA $\gamma$ vector) transformants were used for further phenotyping. For each image of ectomycorrhizal sections $(n=10)$, four types of measurements were obtained using FIJI software (https://fiji.sc), including mantle

230 width, root diameter, Hartig net boundary, and root circumference. These measurements were used to calculate both the ratio of average mantle width to average root diameter and average Hartig net boundary to average root circumference.

\section{Recombinant enzyme production and purification}

235 The methylotrophic yeast Pichia pastoris relying on alcohol oxidase (AOX) to metabolize methanol as its sole carbon source was used as a production host. P. pastoris expression vector $\mathrm{pPICZ} \alpha \mathrm{A}$ contains the $\mathrm{AOX} 1$ promoter for producing heterologous proteins (Ellis et al., 1985, Tschopp et al., 1987, Koutz et al., 1989) and a (His) 6 tag located at the $\mathrm{C}$-terminus for purification. P. pastoris strain X33 was purchased from

240 Invitrogen (Cergy-Pontoise, France). The nucleotide sequence of $L b G H 28 A$ without its signal peptide sequence (Supplemental Information Figure S1) was codon optimized for expression in P. pastoris and synthesized by Genscript (NJ, USA). The synthesized 
245 under the control of the $A O X 1$ promoter. The expression protocol is described in Couturier et al. (2011). Large scale production of the protein was performed in $2 \mathrm{~L}$ nonbaffled flasks, each containing $500 \mathrm{~mL}$ of BMGY medium (1\% yeast extract, $2 \%$ peptone, $1 \%$ glycerol, $400 \mu \mathrm{g} \mathrm{1^{-1 }}$ biotin, and $0.1 \mathrm{M}$ potassium phosphate, $\mathrm{pH} 6.0$ ). P. pastoris was grown overnight at $30^{\circ} \mathrm{C}$ at $200 \mathrm{rpm}$, and recovered by centrifugation

250 the following day when the absorbance was between two and six units. Pellets from five flasks were pooled and resuspended in $100 \mathrm{~mL}$ of BMMY medium (1\% yeast extract, 2\% peptone, $400 \mu \mathrm{g} \mathrm{l}^{-1}$ biotin, $1 \%$ methanol, and $0.1 \mathrm{M}$ potassium phosphate, $\mathrm{pH} 6.0$ ) for each 500-mL flask. Induction was carried out for three days with the addition of three $\mathrm{ml}$ methanol per flask per day. The supernatant was then collected and after 255 setting the $\mathrm{pH}$ to 7.8 with $\mathrm{NaOH} 1 \mathrm{M}$, it was filtered through a $0.22 \mu \mathrm{m}$ filter membrane (Durapore GV membrane filters, $0.22 \mu \mathrm{m}$, Millipore, Molsheim, France). A HisTrap HP column (5 ml, GE Healthcare, France), prepacked with Ni High Performance Sepharose, was connected to an ÄKTA purifier chromatography system (GE Healthcare, France) and equilibrated with the equilibration buffer $(50 \mathrm{mM}$ Tris- $\mathrm{HCl} \mathrm{pH}$ $2607.8,150 \mathrm{mM} \mathrm{NaCl}, 10 \mathrm{mM}$ imidazole) before purification according to the instructions of the manufacturer. The protein was eluted with $50 \mathrm{mM}$ Tris- $\mathrm{HCl}, \mathrm{pH} 7.8,150 \mathrm{mM}$ $\mathrm{NaCl}$ and $250 \mathrm{mM}$ imidazole. Elution was monitored by measuring the absorbance at $280 \mathrm{~nm}$. The fractions corresponding to the eluted protein were pooled, and loaded onto an ultrafiltration column (Vivaspin 3 or 10 kDa MWCO, PES, Sartorius, Palaiseau,

265 France) for concentration and buffer exchange at $4{ }^{\circ} \mathrm{C}$. The proteins were stored at $4{ }^{\circ} \mathrm{C}$ in a $50 \mathrm{mM}$ sodium acetate buffer, $\mathrm{pH}$ 5.2. The concentration of the pure proteins was determined by measuring the absorbance of the solution at $280 \mathrm{~nm}$ on a Nanodrop 2000 (Thermo Fisher Scientific, France) and calculated using Beer's law and the extinction 
coefficient of the protein as determined by ProtParam

270 (http://web.expasy.org/protparam/).

\section{Enzymatic activity assay}

The enzyme activity of LbGH28A was assayed in triplicate by measuring the release of reducing sugars ends from pectin and quantified using the 3-amino-5-nitrosalicylic acid

275 (DNS) method (Couturier et al., 2011). LbGH28A was incubated with the following polysaccharides: pectin of citrus peel (P9135, Sigma Aldrich, France), polygalacturonic acid (Megazyme, Wicklow, Ireland), rhamnogalacturonan (R3875, Sigma Aldrich, France), potato pectic galactan (Megazyme) and lupin galactan (Megazyme). Briefly, $1.53 \mu \mathrm{g}$ of enzyme was mixed with $0.5 \%(\mathrm{w} / \mathrm{v})$ substrate in $50 \mathrm{mM}$ sodium acetate

280 buffer at $\mathrm{pH} 4$ and incubated at $40^{\circ} \mathrm{C}$ for $10 \mathrm{~min}$ in a total volume of $110 \mu \mathrm{l}$. Then, the release of reducing sugars ends was determined using the 3,5-dinitrosalicylic acid (DNS) assay (Sigma). Sixty $\mu \mathrm{l}$ of DNS were added to $60 \mu 1$ of the enzymatic reaction and incubated at $100{ }^{\circ} \mathrm{C}$ for $10 \mathrm{~min}$, and the absorbance at $540 \mathrm{~nm}$ was measured. One unit of enzymatic activity (U) is defined as the amount of enzyme required to release

285 one $\mu \mathrm{mol}$ of glucose reducing-sugar equivalents per minute $\left(\mu \mathrm{mol} . \mathrm{min}^{-1}\right)$ under the defined assay conditions.

For optimal $\mathrm{pH}$ and temperature determination, polygalacturonic acid, $0.5 \%(\mathrm{w} / \mathrm{v})$, was used as substrate. The $\mathrm{pH}$ optimum was determined as described above using 50 $\mathrm{mM}$ sodium acetate with different $\mathrm{pH}\left(3.5,4.0,4.5,5.0\right.$ and 5.5) for $10 \mathrm{~min}$ at $40^{\circ} \mathrm{C}$. For optimal temperature determination, enzyme activity was measured at $30^{\circ} \mathrm{C}, 35^{\circ} \mathrm{C}, 40^{\circ} \mathrm{C}$, $45^{\circ} \mathrm{C}, 50^{\circ} \mathrm{C}$ and $55^{\circ} \mathrm{C}$ for $10 \mathrm{~min}$.

\section{Confocal microscopy and indirect immunofluorescent localization}

Three-week-old ectomycorrhizal rootlets from grey poplar or free-living L. bicolor 
phosphate-buffered saline (PBS) buffer $(137 \mathrm{mM} \mathrm{NaCl}, 2.7 \mathrm{mM} \mathrm{KCl}, 10 \mathrm{mM}$ $\left.\mathrm{Na}_{2} \mathrm{HPO}_{4}, 1.8 \mathrm{mM} \mathrm{K}_{2} \mathrm{HPO}_{4}, \mathrm{pH} 7.4\right)$ at $4^{\circ} \mathrm{C}$. The root segments were embedded in agarose $5 \%(\mathrm{w} / \mathrm{v})$ and cut into $25-30 \mu \mathrm{m}$ radial sections with a VT1200S vibratome (Leica Microsystems, Nanterre, France). Radial sections were sampled from 15

300 ectomycorrhizal rootlets (at $200 \mu \mathrm{m}$ from the root apex) to assess LbGH28A protein accumulation. Sections were retrieved with a brush and carefully transferred onto watch glasses and then were stained according to Felten et al. (2009).

A solution containing $3.5 \mathrm{mg}$ of purified recombinant $\mathrm{LbGH} 28 \mathrm{~A}$ protein was used to elicit the production of polyclonal antibodies in rabbit according to the manufacturer's

305 procedure (Eurogentec, Seraing, Belgium). The indirect immunofluorescent (IIF) localization of the LbGH28A protein was performed by confocal microscopy as described in Zhang et al. (2018). Briefly, sections were transferred onto watch glasses and incubated in 1\% BSA for one hour. Then, BSA was removed and sections were incubated overnight with purified anti-LbGH28A protein rabbit antibody diluted

$3101: 1,000$ in PBS containing $0.5 \%(\mathrm{w} / \mathrm{v}) \mathrm{BSA}$ at $4^{\circ} \mathrm{C}$. The segments were then washed five times in PBS and incubated in the secondary antibody conjugate, a 1:500 dilution of goat anti-rabbit IgG-AlexaFluor 488 conjugate (A-11008, ThermoFisher, France) in PBS for 2 h. After five more washes in PBS, sections were mounted in $80 \%$ glycerol (Merck), 20\% PBS, 5\% w/v propyl gallate (Fluka) and viewed by a Zeiss LSM 800

315 microscope equipped with X10, X40, numerical aperture 1.4. The excitation and emission wavelengths for the Alexa Fluor 488 dye were 500 to $550 \mathrm{~nm}$, respectively. Optical sections were collected at 0.1 to $0.7 \mathrm{~mm}$ intervals with Kalman averaging. As a control, sections were incubated with IgG purified from pre-immune serum diluted to the same concentration as anti-LbGH28A IgG. In addition, we performed a competition

320 epitope binding assay by incubating sections with both anti-LbGH28A protein rabbit antibody diluted 1:1,000 and increasing concentration of recombinant LbGH5-CBM1 protein in PBS containing $0.5 \%(\mathrm{w} / \mathrm{v}) \mathrm{BSA}$ at $4^{\circ} \mathrm{C}$. 
Ultrastructural localization by immunogold labelling

325 Three-week-old ectomycorrhizal root tips or axenic mycelium of L. bicolor S238N were sampled and fixed with $2.5 \%$ glutaraldehyde, $2 \%$ paraformaldehyde in saline phosphate buffer (PBS) for $2 \mathrm{~h}$. The samples were washed in PBS and post-fixed in $1 \%$ osmium tetroxide in distilled water for $1 \mathrm{~h}$, washed and incubated in uranyl acetate $1 \%$ in distilled water overnight at $4^{\circ} \mathrm{C}$. Samples were then dehydrated in $100 \%$ ethanol and

330 acetone, and embedded in Epon resin. Ultrathin sections $(90 \mathrm{~nm})$ were performed on a UC7 Leica Ultra microtome (Leica, Netherlands). Sections on nickel grids were incubated with saturated sodium metaperiodate for $3 \mathrm{~min}$. The grids were washed for 4 min in TBS with $1 \%$ Triton X-100 and then incubated in $10 \%$ normal serum in TBS for $30 \mathrm{~min}$, followed by an overnight incubation with rabbit anti-LbGH28A antibodies

335 (dilution $1 / 5$ ) at $4^{\circ} \mathrm{C}$. The grids were washed in saline Tris buffer (TBS), incubated with secondary antibodies $(6 \mathrm{~nm}$-gold particles linked to anti-rabbit antibodies, Aurion, dilution $1 / 15$ ) for one hour at $37^{\circ} \mathrm{C}$, then washed again in TBS. Grids were incubated for 10 min in $2.5 \%$ glutaraldehyde in $0.05 \mathrm{M}$ cacodylate buffer, washed in water and counter-stained with $1 \%$ uranyl acetate (for $5 \mathrm{~min}$ ) and lead citrate (for $2 \mathrm{~min}$ ),

340 respectively. Acquisitions were performed on a Tecnai G2 electron microscope (FEI, Netherlands) at $200 \mathrm{kV}$. Micrographs were acquired with a Veleta camera (Olympus, Japan) on triplicate samples.

\section{Results}

345 The symbiosis-induced polygalacturonase LbGH28A from L. bicolor

Among the PCWDE genes previously reported as induced in L. bicolor-P. tremulax alba ectomycorrhizal rootlets (Veneault-Fourrey et al., 2014), three genes are annotated as pectinases from the $\mathrm{GH} 28$ family. These $\mathrm{GH} 28$ pectinases are predicted to hydrolyze 
350 the endo-polygalacturonase GH28 subfamily A (Kohler et al., 2015) and has thus been named $L b G H 28 A$. It is induced during the earlier steps of $L$. bicolor-P. tremula $x$ alba symbiosis suggesting it may play a role in plant cell wall remodeling during the Hartig net formation (Veneault-Fourrey et al., 2014). However, the enzymatic activity of LbGH28A has not been confirmed so far. Here, we functionally characterized this

355 LbGH28A gene. The gene contains 2,017 nt with 18 introns, the predicted full-length transcript is 1,065 nt and the deduced polypeptide sequence contains 361 amino-acids (molecular mass (MM) of $37.2 \mathrm{kDa}, \mathrm{pI} 7.61$ ). The LbGH28A polypeptide contains a predicted signal peptide (positions 1 to 16) and GH28 (endo-polygalacturonase) catalytic motif (positions 36 to 344, Pfam 00295) (Supplemental Information Fig. 1). To

360 validate the JGI gene annotation, the full-length $L b G H 28 A \mathrm{cDNA}$ was amplified by RACE PCR from total RNA extracted from ectomycorrhizal rootlets and sequenced (Supplemental Information Fig. 1). The ortholog from L. amethystina (JGI ID \#671067, LaGH28A) is highly similar to LbGH28A (89\% similarity, Supplemental Information Fig. S2), indicating that this symbiosis-induced polygalacturonase is highly conserved within the Laccaria genus. LbGH28A is also orthologous to endopolygalacturonases of the ectomycorrhizal Cortinarius glaucopus, the wood decayer Hypholoma sublateritium and litter decayer Agrocybe pediades (Fig. 1b, see also Supplementary Fig. 10 in Kohler et al., 2015). Interestingly, it shows a low protein sequence similarity (49.5\%) to the symbiosis-induced polygalacturonase of the ascomycetous T. melanosporum.

\section{$\mathrm{LbGH} 28 \mathrm{~A}$ expression is induced by pectin and upregulated in symbiosis}

LbGH28A is a pectin-inducible gene as the presence of Citrus pectin in the growth medium increased its transcription in free-living mycelium by comparison to glucose (Fig. 2A). Polygalacturonic acid also induced the $L b G H 28 A$ expression, but this was not statistically significant. We confirmed by qPCR that $L b G H 28 A$ is transcribed at a lower, 
constitutive level in the free-living mycelium of L. bicolor S238N grown on Pachlewski agar medium (containing $5.5 \mathrm{mM}$ glucose), while it is induced 2.27 -fold in ectomycorrhizal hyphae after three weeks post contact (Fig. 2b). LbGH28A transcripts were not detected in fruiting bodies of L. bicolor (data not shown).

380

LbGH28A expression is critical for the formation of the Hartig net

To confirm the role of the $L b G H 28 A$ gene in ectomycorrhiza establishment, we generated a series of transgenic L. bicolor lines with a lowered level of $L b G H 28 A$ by knocking down its expression by RNAi-mediated gene silencing. Four randomly selected L. bicolor mutant strains (LbGH28 RNAi-1, LbGH28 RNAi-2, LbGH28 RNAi3, LbGH28 RNAi-4) and empty vector controls (EV7 and EV9) were co-cultured with poplar seedlings to establish ectomycorrhiza. The $L b G H 28$ RNAi-3 and $L b G H 28$ RNAi-4 mutants were significantly impaired in their ability to form ectomycorrhizas (Fig. 3a) by comparing to empty vector controls. While the percentage of 390 ectomycorrhizal root tips was $57 \%$ and $53 \%$ for the EV controls, it reached only $11 \%$ and $16 \%$ for the $L b G H 28$ RNAi-3 and $L b G H 28$ RNAi-4 mutants, respectively. The rate of ectomycorrhiza formation by the $L b G H 28$ RNAi-1 (53 \%) and $L b G H 28$ RNAi-2 (50 \%) transformant lines was similar to the EV controls. To assess the development of symbiotic structures in planta, we harvested colonized root tips and measured the mantle sheath thickness and the Hartig net depth after staining of hyphae by using wheat germ agglutinin (WGA) conjugated with Alexa Fluor 488 (Fig. 4). The laserscanning confocal microscopy images showed that the $L b G H 28 A$ RNAi mutant lines have a significantly reduced mantle sheath and Hartig net (Fig. 3c,d). The mantle thickness was $19.5 \pm 0.9 \mu \mathrm{m}$ and $18.2 \pm 2.6 \mu \mathrm{m}$ per root section for the empty vector controls, while it ranged from $10.4 \pm 0.7 \mu \mathrm{m}$ to $21.7 \pm 1.3 \mu \mathrm{m}$ for the $L b G H 28 A$ RNAi mutant lines. These values were significantly lower for the $L b G H 28$ RNAi-2, $L b G H 28$ RNAi-3 and LbGH28 RNAi-4 mutants ( $\mathrm{P}<0.01$, student's test, $\mathrm{n}>150)$, despite the 
substantial heterogeneity observed between ectomycorrhizal root tips. The Hartig net depth was $8.9 \pm 0.2 \mu \mathrm{m}$ and $11.6 \pm 0.5 \mu \mathrm{m}$ per root section for the empty vector controls, whereas it was significantly lower for the $L b G H 28 A$ RNAi mutant lines $(\mathrm{P}<0.01$, student's test, $\mathrm{n}>150)$, ranging from $4.4 \pm 0.2 \mu \mathrm{m}$ to $8.9 \pm 0.4 \mu \mathrm{m}$. Taking together, these morphometric measurements suggest that $L b G H 28 \mathrm{~A}$ facilitates the fungal ingress into the host intercellular space and as a consequence, participates to the symbiosis establishment.

410

Cloning, expression and purification of $\mathrm{LbGH} 28 \mathrm{~A}$ in $P$. pastoris

LbGH28A, without its native signal peptide, was successfully expressed in P. pastoris and purified by affinity chromatography enabling its biochemical characterization. SDSPAGE showed that the recombinant LbGH28A migrated as a single band with a

415 molecular mass (MM) of $55 \mathrm{kDa}$ (Fig. 5a). This MM was higher than the predicted MM $(37.2 \mathrm{kDa})$ suggesting that the mature protein synthesized in yeast was glycosylated. The purified recombinant LbGH28A protein was then used to assess the enzyme activity and produce polyclonal antibodies in rabbit.

420 Enzymatic properties of $\mathrm{LbGH} 28 \mathrm{~A}$

The substrate specificity of the recombinant LbGH28A was assayed by using Citrus pectin, polygalacturonic acid, rhamnogalacturonan, galactan or pectic galactan. LbGH28A exhibits an endo-polygalacturonase activity, as indicated by the increase of released reduced sugars from Citrus pectin and polygalacturonic acid (Fig. 5b). No 425 activity was observed on rhamnogalacturonan, galactan or pectic galactan (Fig. 5b). The optimal $\mathrm{pH}$ and temperature of $\mathrm{LbGH} 28 \mathrm{~A}$ are 4.5 and $35^{\circ} \mathrm{C}$, respectively (Fig. 5c,d).

LbGH28A is localized on fungal and plant cell walls 
430 ectomycorrhizal rootlets to localize LbGH28A. Probing ectomycorrhizal root sections with anti-LbGH28A antibodies led to an intense labeling of hyphae constituting the mantle and the Hartig net (Fig. 6a). Labeling was mainly detected at the periphery of the hyphae and coincided with the cell wall chitin labeled by wheat germ agglutinin (WGA633), supporting a cell wall and/or apoplastic localization. In the presence of the recombinant LbGH28A (competitive assay), the binding of antibodies to LbGH28A was precluded and no signal was detected, confirming the high specificity of the immune serum (Fig. 6b). No labelling was detected when a pre-immune serum was used instead of LbGH28A-antibody (Fig. 6c).

Immunocytolocalization of $\mathrm{LbGH} 28 \mathrm{~A}$ was performed on ultrathin sections of

440 L. bicolor free-living mycelium and L. bicolor-P. tremula $x$ alba ectomycorrhizal rootlets by transmission electronic microscopy (TEM) (Fig. 7). At the tip of the symbiotic hyphae penetrating between epidermal cells, the $6 \mathrm{~nm}$-gold particles, indicating the presence of LbGH28A, were detected in the merged fungal and plant cell walls of the symbiotic interface (Fig. 7cd). This localization confirms that LbGH28A is

445 secreted in the symbiotic interface where it bounds to fungal and plant cell wall materials. No labelling was detected when a pre-immune serum was used instead of LbGH28A-antibody (Fig. 7ef). In addition, no labelling was observed in the plant cell walls of non-mycorrhizal poplar rootlets when pre-immune and immune sera were used (Fig. 7ghij), confirming that the anti-LbGH28A antibody is specific to the fungal

450 LbGH28A with no cross-hybridization to poplar polygalacturonase(s). LbGH28A was also detected in the cell walls of the free living mycelium (Fig. 7ab), despite the low expression of LbGH28A (Fig. 2b). Finally, we also immunolocalized the symbiosisinduced ß-1,4-endoglucanase LbGH5-CBM1 from L. bicolor (Zhang et al., 2018) in the ectomycorrhizal root tips used for the LbGH28A immunolocalization. LbGH5-CBM1 was strictly located in fungal cell walls (Fig. 8). 


\section{Discussion}

Colonization of the root middle lamella space by ectomycorrhizal hyphae is required for the formation of the Hartig net, the symbiotic interface, and for subsequent symbiosis

460 development. This fungal penetration between epidermal and cortical root cells leads to biochemical changes in both hyphal and root cell walls (Balestrini \& Kottke, 2016).

These changes include localized loosening and swelling of cell walls, and redistribution of un-esterified pectins (Balestrini et al., 1996; Balestrini \& Bonfante, 2014; Sillo et al., 2016). Fungal ingress in the middle lamella is thought to rely on the mechanical force

465 that results from hyphal tip growth (Peterson \& Massicotte, 2004), the effect of auxins released by colonizing hyphae (Gay et al., 1994 a,b) and the activity of fungal PCWDEs (Veneault-Fourrey et al., 2014; Sillo et al., 2016). Although the arsenal of PCWDEs encoded by L. bicolor and other ectomycorrhizal fungi is restricted by comparison to saprotrophic fungi (Martin et al., 2008; Kohler et al., 2015; Miyauchi et al., 2020),

470 several of the remaining PCWDE genes are induced in ectomycorrhizal associations (Martin et al., 2008, 2010; Kohler et al., 2015; Peter et al., 2016; Sillo et al., 2016; Miyauchi et al., 2020). Among the $>20$ symbiosis-upregulated PCWDEs of L. bicolor, endoglucanases (GH5), pectin methyl esterases (CE8), polygalacturonases (GH28) and cellulose acting-lytic polysaccharide monooxygenases (LPMO, AA9) are induced at

475 different stages of ectomycorrhiza establishment (Veneault-Fourrey et al., 2014), suggesting that plant cell wall remodeling through pectin and cellulose modifications may be involved in Hartig net development. We previously reported that the symbiosisinduced ß-1,4-endoglucanase LbGH5-CBM1 from L. bicolor, acting on poplar cellulose, mannans and galactomannan, is involved in cell wall remodeling during 480 formation of the Hartig net and this enzyme is required for successful symbiotic fungal colonization of poplar rootlets (Zhang et al., 2018). Also L. bicolor ß-glucanase GH131, active on polysaccharides with $\beta-1,4, \beta-1,3$, and mixed $\beta-1,4 / 1,3$ glucosidic linkages, is 
induced upon ectomycorrhiza formation (Anasontzis et al., 2019), further strengthening the concept of fungal PCWDEs as fundamental factors in the establishment of symbiotic plant-fungus interactions.

In the present study, we functionally characterized the symbiosis-upregulated LbGH28A from L. bicolor and confirmed its role in ectomycorrhiza formation by RNAi knock-down of the $L b G H 28 A$ gene. A recombinant protein was produced and used for assaying the enzymatic activity of LbGH28A. These studies confirmed that LbGH28A

490 is an endopolygalacturonase of family GH28. Finally, we localized the enzyme in L. bicolor cell walls and ectomycorrhizal interface by indirect immunofluorescence confocal microscopy and immunogold cytolocalization.

As showed by the phylogenetic analysis, this endopolygalacturonase is closely related to GH28A polygalacturonases from saprotrophic fungi. This suggests similar substrate specificities for these enzymes, namely activity on pectins in plant cell walls and plant residues, as well as a common evolutionary origin in Agaricomycotina. Transcription of $L b G H 28 A$ in the free-living mycelium was induced by pectin, suggesting that its activity is indeed related to pectin metabolism. This was further confirmed by studies on the recombinant LbGH28A protein. The enzyme displays 500 typical polygalacturonase activity and as expected, cannot hydrolyzes rhamnogalacturonans and galactans, which do not contain any polygalacturonic acid. Moreover, LbGH28A showed an optimal activity at $\mathrm{pH} 4$ to 5, values similar to those of the plant apoplastic space. In addition, this degradative activity is enough to support the growth of L. bicolor mycelium on pectin as a sole carbon source (Fig. S1 in Veneault-

505 Fourrey et al., 2014). Pectin methylation is an important factor influencing the activity of polygalacturonases (Lionetti et al., 2017). It remains to determine whether the symbiosis-induced pectin methylesterases (CE8) of L. bicolor act synergistically with LbGH28A to modify host pectins at the symbiotic interface. 
Indirect immunofluorescence confocal microscopy using anti-LbGH28 antibodies

510 showed that LbGH28A is localized at the periphery of L. bicolor hyphae forming the mantle sheath and the Hartig net. Its co-localization with/or adjacent to chitin label supports an extracellular/cell wall localization for this enzyme. Finally, immunogold cytolocalization microscopy confirmed that LbGH28A accumulates at the surface of both fungal and plant cells walls, i.e. the symbiotic interface, at the hyphal front

515 penetrating between the epidermal root cells, while the LbGH5-CBM1 was mainly detected in the fungal cell walls.

Although L. bicolor lacks GH6 and GH7 cellobiohydrolases (Martin et al., 2008), the endocellulase LbGH5-CBM1 (Zhang et al., 2018), ß-glucanase GH131 (Anasontzis et al., 2019), expansins (Veneault-Fourrey et al., 2014) and here characterized

520 polygalacturonase LbGH28A are likely involved in the limited alteration/remodelling of the host cell walls, thereby facilitating root penetration and Hartig net differentiation. We speculate that cellulose-acting LPMO (AA9) and GH5-CBM1 are involved in the loosening of the cellulose microfibrils and LbGH28A in remodeling the middle lamella through pectin (homogalacturonan) hydrolysis. However, it remains to be assessed how

525 the mechanical cell wall properties, such as loosening and softening, and the strength and extension of adhesion areas between adjacent epidermal cells are altered during hypae colonization. These plant cell wall modifications may involve the depolymerization of matrix glucans and pectins, the pectin solubilization and removal of neutral sugars from pectin side chains. Such changes are expected to weaken the host

530 cell walls and increase cell separation, which in combination with the turgor pressure of the hyphal tip, bring about textural changes. Atomic force microscopy (Zhang et al., 2016) will be used to further characterize the nanostructure of plant cell wall polysaccharides during ectomycorrhiza development. We speculate that these subtle modifications in the cell wall structures do not trigger the host plant immune response.

535 Even if a moderate plant immune response is activated by cell wall elicitors, 
mycorrhiza-induced small effectors, such as the L. bicolor MiSSP7 and MiSSP7.6, would be able to dampen the defense reactions (Martin et al., 2016).

\section{Acknowledgments}

540 The research was funded by the French Research National Agency (ANR-14-CE060020, project FUNTUNE), the Laboratory of Excellence Advanced Research on the Biology of Tree and Forest Ecosystems (ARBRE; ANR-11-LABX 0002 01, project SYMWOOD), the Genomic Science Program, US Department of Energy, Office of Science, Biological and Environmental Research as part of the Plant-Microbe Interfaces

545 Scientific Focus Area (http://pmi.ornl.gov), and by grants from Universidad Nacional de Quilmes, Consejo Nacional de Investigaciones Científicas y Técnicas, and Agencia Nacional de Promoción Científica y Tecnológica; and by grants from National Natural Science Foundation of China (31901279), Natural Science Foundation of Gansu Province (20JR5RA276), and Fundamental Research Funds for the Central Universities

550 (lzujbky-2019-ct01). The immunogold cytochemical electron microscopy was performed on the PiCSL-FBI core facility (IBDM, AMU-Marseille), a member of the France-BioImaging national research infrastructure (ANR-10-INBS-04). We would like to thank Dr. Raffaella Balestrini (University of Torino, Italy) for a gift of WGA.

\section{Author contributions}

F.M. and J-G.B. planned and designed the research. F.Z., A.L., N.B.; M.H.; M.K., and A.D. performed experiments. F.Z., G.E.A., A.P., C.V-F., A.K., M-N.R., B.H., J-G.B. and F.M. analyzed the data. F.M. and F.Z. wrote the manuscript with the help of the coauthors.

\section{ORCID}

Annegret Kohler: http://orcid.org/0000-0002-9575-9567 
bioRxiv preprint doi: https://doi.org/10.1101/2021.09.24.461608; this version posted September 24,2021 . The copyright holder for this preprint (which was not certified by peer review) is the author/funder, who has granted bioRxiv a license to display the preprint in perpetuity. It is made available under aCC-BY-NC-ND 4.0 International license.

Jean-Guy Berrin: http://orcid.org/0000-0001-7570-3745

Francis Martin: http://orcid.org/0000-0002-4737-3715

565 Zhang Feng: https://orcid.org/0000-0002-5039-2124 


\section{References}

Anasontzis GE, Lebrun M, Haon M, Champion C, Kohler A, Lenfant N, Martin F, O'Connell RJ, Riley R, Grigoriev IV et al. 2019. Broad-specificity GH131 $\beta$ glucanases are a hallmark of fungi and oomycetes that colonize plants. Environ Microbiol 21: 2724-2739.

Balestrini R, Bonfante P. 2014. Cell wall remodeling in mycorrhizal symbiosis: a way towards biotrophism. Front Plant Sci 5: 237.

Balestrini R, Kottke I. 2016. Molecular mycorrhizal symbiosis. John Wiley \& Sons, Inc 47-61.

Balestrini R, Hahn MG, Faccio A, Mendgen K, Bonfante P. 1996. Differential localization of carbohydrate epitopes in plant cell walls in the presence and absence of arbuscular mycorrhizal fungi. Plant Physiol 111: 203-213.

Brundrett MC. 2002. Coevolution of roots and mycorrhizas of land plants. New Phytologist 154: 275-304.

Christiaens S, Van Buggenhout S, Houben K, Jamsazzadeh Kermani Z, Moelants KR, Ngouémazong ED, Van Loey A, Hendrickx ME. 2016. Process-structurefunction relations of pectin in food. Crit Rev Food Sci Nutr. 56:1021-42.

\section{Couturier M, Feliu J, Haon M, Navarro D, Lesage-Meessen L, Coutinho PM,} Berrin JG. 2011. A thermostable GH45 endoglucanase from yeast: impact of its atypical multimodularity on activity. Microb Cell Fact 10: 103.

Dietz S, von Bülow J, Beitz E, Nehls U. 2011. The aquaporin gene family of the ectomycorrhizal fungus Laccaria bicolor: lessons for symbiotic functions. New Phytologist 190: 927-940.

590 Ellis SB, Brust PF, Koutz PJ, Waters AF, Harpold MM, Gingeras TR. 1985. Isolation of alcohol oxidase and two other methanol regulatable genes from the yeast Pichia pastoris. Mol. Cell. Biol 5:1111-1121. 
Felten J, Kohler A, Morin E, Bhalerao RP, Palme K, Martin F, Ditengou FA, Legue V. 2009. The ectomycorrhizal fungus Laccaria bicolor stimulates lateral root formation in poplar and Arabidopsis through auxin transport and signaling. Plant Physiol 151: 1991-2005.

Gay G, Normand L, Marmeisse R, Sotta B, Debaud JC. 1994. Auxin overproducer mutants of Hebeloma cylindrosporum Romagnesi have increased mycorrhizal activity. New Phytologist 128: 645-657.

600 Gea L, Normand L, Vian B, Gay G. 1994. Structural aspects of ectomycorrhiza of Pinus pinaster (Ait.) Sol. formed by an IAA-overproducer mutant of Hebeloma cylindrosporum Romagnési. New Phytologist 128: 659-670.

Kemppainen MJ, Pardo AG. 2010. pHg/pSILBAgamma vector system for efficient gene silencing in homobasidiomycetes: optimization of ihpRNA - triggering in the mycorrhizal fungus Laccaria bicolor. Microb Biotechnol 3: 178-200.

Kohler A, Kuo A, Nagy LG, Morin E, Barry KW, Buscot F, Canback B, Choi C, Cichocki N, Clum A et al. 2015. Convergent losses of decay mechanisms and rapid turnover of symbiosis genes in mycorrhizal mutualists. Nat Genet 47: 410-415.

Koutz P, Davis GR, Stillman C, Barringer K, Cregg J, Thill G. 1989. Structural comparison of the Pichia pastoris alcohol oxidase genes. Yeast 5: 167-177.

Laurent P, Voiblet C, Tagu D, De Carvalho D, Nehls U, De Bellis R, Balestrini R, Bauw G, Bonfante P, Martin F. 1999. A novel class of ectomycorrhiza-regulated cell wall polypeptides in Pisolithus tinctorius. Mol Plant Microbe In 12: 862-871.

Martin F, Aerts A, Ahren D, Brun A, Danchin EGJ, Duchaussoy F, Gibon J, Kohler A, Lindquist E, Pereda V et al. 2008. The genome of Laccaria bicolor provides insights into mycorrhizal symbiosis. Nature 452: 88-92.

Martin F, Kohler A, Murat C, Balestrini R, Coutinho PM, Jaillon O, Montanini B, Morin E, Noel B, Percudani R et al. 2010. Perigord black truffle genome uncovers evolutionary origins and mechanisms of symbiosis. Nature 464: 1033-1038. 
620 Martin F, Kohler A, Murat C, Veneault-Fourrey C, Hibbett DS. 2016. Unearthing the roots of ectomycorrhizal symbioses. Nature Reviews Microbiology 14: 760-773.

Massicotte HB, Peterson RL, Melville LH. 1989. Hartig Net structure of ectomycorrhizae synthesized between Laccaria bicolor (Tricholomataceae) and two hosts: Betula alleghaniensis (Betulaceae) and Pinus resinosa (Pinaceae). Am. J. Bot 76: $1654-1667$.

Miyauchi S, Kiss E, Kuo A, Drula E, Kohler A, Sánchez-García M, Morin E, Andreopoulos B, Barry KW, Bonito G, et al. 2020b. Large-scale genome sequencing of mycorrhizal fungi provides insights into the early evolution of symbiotic traits. Nature Communications 11: 5125.

630 Pachlewski, R. 1967. "Mikotrofizm systemu korzeniowego," in Zarys Fizjologii Sosny Zwyczajnej, eds S. Bialobok, and W. Zelawski, (Poznan: PWN Warszaw).

Peterson RL, Massicotte HB. 2004. Exploring structural definitions of mycorrhizas, with emphasis on nutrient-exchange interfaces. Canadian Journal of Botany 82: 1074-1088.

635 Peter M, Kohler A, Ohm RA, Kuo A, Krützmann J, Morin E, Arend M, Barry KW, Binder M, Choi C. et al. 2016. Ectomycorrhizal ecology is imprinted in the genome of the dominant symbiotic fungus Cenococcum geophilum. Nat. Commun. 7, 12662.

Pitarch A, Sánchez M, Nombela C, Gil C. 2002. Sequential fractionation and two640 dimensional gel analysis unravels the complexity of the dimorphic fungus Candida albicans cell wall proteome. Mol Cell Proteomics 1: 967-982.

Pfaffl, MW. 2001. A new mathematical model for relative quantification in real-time RT-PCR. Nucleic Acids Research 29: e45.

Read DJ, Leake JR, Perez-Moreno J. 2004. Mycorrhizal fungi as drivers of ecosystem processes in heathland and boreal forest biomes. Canadian Journal of Botany 82: 1243-1263. 
Smith SE, Read D. 2008. Mycorrhizal Symbiosis (Third Edition). London: Academic Press.

Tagu D, De Bellis R, Balestrini R, De Vries OMH, Piccoli G, Stocchi V, Bonfante

P, Martin F. 2001. Immunolocalization of hydrophobin HYDPt-1 from the ectomycorrhizal basidiomycete Pisolithus tinctorius during colonization of Eucalyptus globulus roots. New Phytologist 149: 127-135.

Tschopp JF, Brust PF, Cregg JM, Stillman CA, Gingeras TR. 1987. Expression of the lacZ gene from two methanol-regulated promoters in Pichia pastoris. Nucleic Acids Research 15: 3859-3876.

van der Heijden MG, Martin FM, Selosse MA, Sanders IR. 2015. Mycorrhizal ecology and evolution: the past, the present, and the future. New Phytol 205: 14061423.

Veneault-Fourrey C, Commun C, Kohler A, Morin E, Balestrini R, Plett J,

Danchin E, Coutinho P, Wiebenga A, de Vries RP et al. 2014. Genomic and transcriptomic analysis of Laccaria bicolor CAZome reveals insights into polysaccharides remodelling during symbiosis establishment. Fungal Genet Biol 72: $168-181$.

Zhang T, Zheng Y, Cosgrove DJ. 2016. Spatial organization of cellulose microfibrils and matrix polysaccharides in primary plant cell walls as imaged by multichannel atomic force microscopy. Plant J. 8:179-92.

Zhang F, Anasontzis GE, Labourel A, Champion C, Haon M, Kemppainen M, Commun C, Deveau A, Pardo A, Veneault-Fourrey C et al. 2018. The ectomycorrhizal basidiomycete Laccaria bicolor releases a secreted $\beta-1,4$ endoglucanase that plays a key role in symbiosis development. New Phytol. 220: 1309-1321.

Zhang T, Tang H, Vavylonis D, Cosgrove DJ. 2019. Disentangling loosening from softening: insights into primary cell wall structure. Plant J. doi:10.1111/tpj.14519 
bioRxiv preprint doi: https://doi.org/10.1101/2021.09.24.461608; this version posted September 24, 2021. The copyright holder for this preprint (which was not certified by peer review) is the author/funder, who has granted bioRxiv a license to display the preprint in perpetuity. It is made available under aCC-BY-NC-ND 4.0 International license. 


\section{Legends of Figures}

Figure 1. The symbiosis-induced endopolygalacturonase LbGH28A from

L. bicolor. (a) LbGH28A modular structure showing the putative signal peptide (in

680 pink) and the catalytic GH28 domain (in green). (b) Phylogenetic relationship of LbGH28A to other related GH28 polygalacturonases in Agaricomycotina. The phylogenetic neighbor-joining tree was deduced from the alignment of fungal GH28 proteins using MAFFT (version 7) with the consistency-based method. The tree was constructed using FastTree (version 2.1.11). and MEGAX (version 10.1.8).

685

Figure 2. $L b G H 28 A$ expression is induced by polygalacturonate and pectin, and upregulated in ectomycorrhiza. (a) $L b G H 28 A$ relative expression in $L$. bicolor freeliving mycelium grown on $0.5 \%$ glucose, $0.5 \%$ polygalacturonic acid or $0.5 \%$ Citrus pectin as a sole source of carbon. (b) Relative $L b G H 28 A$ expression in wild-type S238N

690 (WT) in free-living mycelium and ectomycorrhizal root tips assayed by quantitative RTPCR. Data presented are the means of three independent biological replicates with two technical replicates per each fungal strain. Error bars represent standard deviation $( \pm \mathrm{SD})$

\section{Figure 3. Rate of formation and morphometric features of ectomycorrhizas} established by $L b G H 28 A$ RNAi-silenced- and control strains. (a) Percentage of ectomycorrhizal rootlets formed by the empty vector control strains (EV-7, EV-9) and RNAi-silenced lines (LbGH28 RNAi-1, LbGH28 RNAi-2, LbGH28 RNAi-3, LbGH28 RNAi-4) three weeks after contact. For each image of ectomycorrhizal sections (b), morphometric measurements (i.e., Hartig net depth (c) and mantle sheath thickness (d)) were carried out using the FIJI software (https://fiji.sc). (b) A section of ectomycorrhizal roots showing how the mantle sheath thickness and Hartig net depth were measured. 
Values are shown for the rate of ectomycorrhiza formation (a), the mantle sheath thickness (c) and the Hartig net depth (d). Bars represent the mean of the data and error 705 bars represent the SE of the mean. **, $P<0.01$, ***, $P<0.001$, student's $t$ test.

Figure 4. $L b G H 28 A$ is required for Hartig net formation. Representative transverse cross sections of Populus roots colonized by (a-b) empty vector (EV-7 and EV-9) control strains or (c-f) RNAi-silenced (LbGH28 RNAi-1, LbGH28 RNAi -2, LbGH28

710 RNAi -3, $L b G H 28$ RNAi -4) strains sampled three weeks after contact. Colonized roots were sectioned and stained with WGA conjugated with Alexa Fluor 488 (green) and propidium iodide (red) and imaged on a confocal laser scanning microscope. M, Mantle; HN, Hartig net; EC, epidermal cells; CC, cortical cells. Scale bar, $50 \mu \mathrm{m}$

715 Figure 5. Heterologous production and enzymatic activity of LbGH28A. (a) SDSPAGE analysis of the purified recombinant protein LbGH28A (5 $\mu \mathrm{g})$. (b) Specific enzyme activity on different substrates. (c) Effect of $\mathrm{pH}$ and (d) temperature on LbGH28A specific enzyme activity. Hydrolysis of polygalacturonic acid ( $\left.5 \mathrm{mg} \mathrm{ml}^{-1}\right)$ by the recombinant LbGH28A was measured in $50 \mathrm{mM}$ sodium acetate buffer for $10 \mathrm{~min}$.

720 MM, molecular mass markers (15 to $180 \mathrm{kDa}$ ). Data presented are the means of three independent replicates. Error bars represent standard deviation ( \pm SD).

Figure 6. Immunolocalization of LbGH28A in Populus tremula $x$ alba-L. bicolor ectomycorrhizal rootlets. All images were obtained by using indirect

725 immunofluorescence confocal laser microscopy. (a) Transversal sections of 3-week-old ectomycorrhizas stained for LbGH28A with anti-LbGH28A immune serum (green), for fungal cell wall chitin with WGA633 (red), bright field and the overlaid images. (b), A pre-immune rabbit serum was used instead of anti-LbGH28A immune serum. (c) Binding of anti-LbGH28A antibodies to LbGH28A was blocked by pre-incubating 
730 sections in the presence of the recombinant LbGH28A protein confirming the binding specificity. Abbreviations: M, fungal mantle; HN, Hartig net; EC, epidermal cell; CC, cortical cell. Scale bar, $20 \mu \mathrm{m}$.

Figure 7. Immunogold cytolocalization of LbGH28A at the symbiotic interface of

735 ectomycorrhizal root tips. TEM images showed the $6 \mathrm{~nm}$-gold particles corresponding to the localization of the LbGH28A antibody/LbGH28A protein in the free-living mycelium of L. bicolor (a, b) or L. bicolor hyphal tips (HT) colonizing the middle lamella/apoplastic space (AS) between two epidermal cells (EC) of P. tremula x alba roots $(\mathbf{c}, \mathbf{d})$. No labelling was observed when sections were incubated without antibody

$740 \quad(\mathbf{e}, \mathbf{f})$; no labelling was observed in cell walls of non-mycorrhizal rootlets incubated with LbGH28A antibody $(\mathbf{g}, \mathbf{h})$ or without antibody as control $(\mathbf{i}, \mathbf{j})$. Ultra-thin transverse sections of 3-week-old ectomycorrhizas or poplar roots were incubated with antiLbGH28A rabbit antibodies coupled to $6 \mathrm{~nm}$ gold particles. Gold particles indicating LbGH28A localization are highlighted by black arrows. PCW; plant cell wall, FCW; 745 fungal cell wall, Hy, fungal hyphae.

Figure 8. Immunogold cytolocalization of LbGH5-CBM1 in ectomycorrhiza and free-living mycelium of $\boldsymbol{L}$. bicolor. TEM images showing the localization of the LbGH5-CBM1 antibody/LbGH5-CBM1 protein in L. bicolor hyphal tips (Hy)

750 colonizing the middle lamella/apoplastic space between two epidermal cells of P. tremula $x$ alba roots. Ultra-thin transverse sections of 3-week-old ectomycorrhizas were incubated with anti- LbGH5-CBM1 rabbit antibodies (Zhang et al., 2018) coupled to gold particles. Gold particles indicating LbGH5-CBM1 localization are highlighted by black arrows. PCW; plant cell wall, FCW; fungal cell wall, Hy, fungal hyphae. 
Supporting Information Table S1. List of primer sequences used in this study.

\begin{tabular}{ll}
\hline Name of primer & Sequence $\left(\mathbf{5}^{\prime}\right.$ - $\left.\mathbf{3}^{\prime}\right)$ \\
\hline GH28AGSP1-F & TATTGGGATGGTACGGGTCTCAGTTCC \\
GH28AGSP1-R & AATGCCATTGCTCCTGTTATTGGCGTAG \\
GH28AGSP2-F & CGGCCATGACGTAACGATTCAGAACTG \\
GH28AGSP2-R & AGAGCCTGGTCACTGTCGGTGATGATG \\
GH28A-RNAi-NF-SalI & TAGTCGACCACAATTACACTTCGGGGCT \\
GH28A-RNAi-NR-HindIII & TTAAGCTTGCAAGGCAATCGTCTTGATT \\
GH28A-RNAi-NF-StuI & ATAGGCCTCACAATTACACTTCGGGGCT \\
GH28A-RNAi-NR-BglII & TTAGATCTGCAAGGCAATCGTCTTGATT \\
GH28A-qPCR-F2 & GCAAAGCAGGTTGCGGTAAA \\
GH28A-qPCR-R2 & CAGTATGTTCGCGTTGCTGG \\
$446085-F$ & CAAAGCCGCCTACGCCTAAA \\
$446085-R$ & GGCATAGTGCCTGCATCGAG \\
$609242-\mathrm{F}$ & CGCGGATCAGGGTCTCTTTT \\
$609242-\mathrm{R}$ & TGGGACCCTGTCTGAATGCT \\
$611151-\mathrm{F}$ & TGGTTTTGCGGCGTTCTTCT \\
$611151-\mathrm{R}$ & TATGTCGTGCCCTGTCCCAA \\
$319764-\mathrm{F}$ & GTTCAAAGCATCCCCAAGC \\
$319764-\mathrm{R}$ & CAACCACCGAAACCGTAGAG \\
\hline
\end{tabular}




\section{Supplementary Figures}

Figure S1. The L. bicolor GH28A polygalacturonase. (a) Amino acid sequence highlighting the native signal peptide (underlined), the putative $\mathrm{N}$-glycosylation sites (in blue) and the conserved catalytic residues (in red); (b) PFAM domain (pfam00295) motif; (c) genomic sequence with 19 exons (in red) and 18 introns (in black); (d) coding (CDS) sequence; (e) nucleotide sequences of $L b G H 28 \_A$ promoter and (f) the nucleotide sequence of the RNAi target. Signal peptide is underlined and the catalytic domain is displayed in green.

\section{(a)}

MLSVFSVLLFAGVVLACQGSISSLSDVDLAVKCTTITLRGFTVPAGQGLILNLLPGTTVTMAGD ISFGNKSWAGPLFTIIGTSITFNGNGHNFNGGGPFYWDGTGLSSGTQKPDPMMRIRMSGTFONV TVVNSPARVFSVSNPAPLLMTKLTVDNSOGDYPNNRSYGTAAGHNTDGFDVSGHDVTIONCTVH NQDDCLAIGKGTNI IFDRNTCIGGHGISIGSIGSNVAVSGVIVTGNIITDSDQALRIKTIASAT GSVVGNVTYSGNTAKGIRRFGVIIDQSYPSTLGTPGTGTIISNVNFGSPTSNIAVNSGAKQVAV NCGYGSCTGTWDWHYLSVKGGVTSPPSNANILGLMGNMLSV

\section{(b)}

Pssm-ID: 306744 Cd Length: 321 Bit Score: 196.84 E-value: $2.08 \mathrm{e}-60$

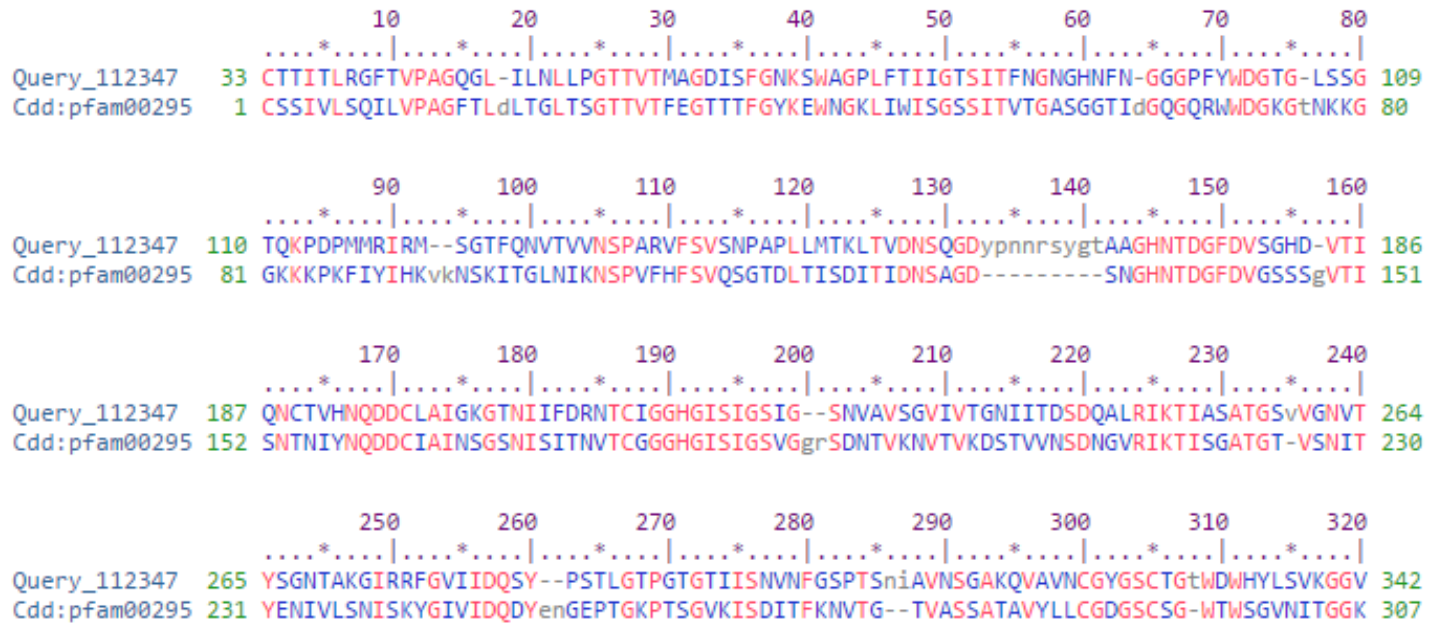


(C)

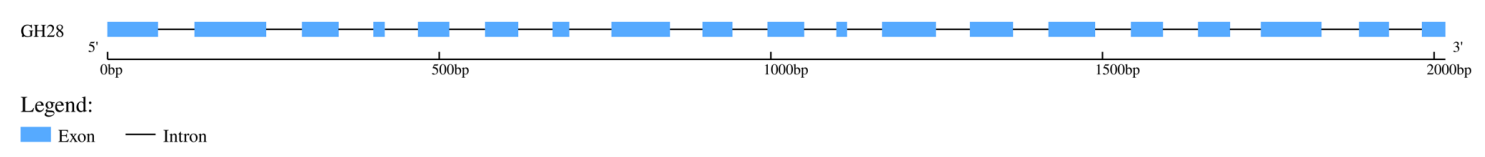

AAGAGAAGACCTGAATGGAGGAACGCCTACTGCTTAGCGCCATTTACAAGGCGCTACTGTAGCAACCCAACTTGTGTTATTGGTTTGA ATTCTATGCTTGAAGTGCTCATGACGACAGAGTTTTGATGTCCGGAAGTCATTTATCCGAATTCTCTGCGATTCTTGGATCTGGCGAG АTCCCTCCACTAATCTCTTCCTCCGAGCGGCCAATTAACTCAACTTGAGGAGATATAAATGCTCGAGGGAAACTTTGGCGACATTCAA CATGCTCTCTGTTTTCTCCGTGTTGCTCTTCGCTGGTGTAGTGCTAGCATGCCAGGGGTCCATATCGTCTTTGTCTGGTGAAGTGCTG CGTTACGATCTCCTCCAACCCATCTGAATATAGAAAACGAAAAGACGTGGATTTAGCTGTCAAGTGCACCACAATTACACTTCGGGGC TTCACTGTTCCTGCTGGCCAGGGTTTAATTCTGAATCTTTTGCCAGGAACGACGGTTACGATGGGTAGCTCAAATACATTCTACTACG ATTCATCAGCTTATTTCACCCTTATTATAGCTGGTGATATTTCATTTGGCAACAAAAGCTGGGCAGGCCCCCTTTTCACCATAATGTA GGCGAATTTCTCCAATGCAAGCCCACCTTGATTGAGTAACATTTCTTTAGTGGAACTTCTATCACATGTGCGTATTTCCATACGCCTT GGACAAGTTAAAGTTGAATTCTGCAATAGTCAACGGGAACGGCCATAATTTCAATGGTGGAGGTCCCTTCTATTGGGTAAAATCGATT ACGGAAGTAAAATACCTTTGAACGCTAAACGACTTCGTTTAGGATGGTACGGGTCTCAGTTCCGGCACACAAAAGCCAGATCCTATGA TGAAGTTTGCTCTGTGTTGCTTATCTTTCCGACGAGTTCTCACTGTCTTCTGGCAGGATCGAAATGTCAGGAATTTTTCAGGTCAGTT TTCTTGCCTCTTTGGCATACATCCAAACTTCTGAATGACTGCTAACACATACTTTAGAATGTAACAGTGGTCAACTCGCCCGCTCGGG СTTTCGCTGTGTCAAATCCCGCACCCTTGTTGATGACAGGATTGACAGTTGATGACTGTAGGAGGCATATAATTTATTCCACGACTAT CCATTCAATCGTTTATAGCTCAAGGAGACTACGCCAATAACAGGAGCAATGGCATTACCGCTGGTAGGCTCGTTCAACTTCGTAAACA GAGATGGACAATCCATCCATCCATTCAGGCCACAACACCGATGGATTCGACGTGAGCGGCCATGACGTAACGATTCAGAACTGGTATG AAGACTCAATGAAATGATGGGTACTTTCGCTTACTATTTCCTAGTACGGTTCATAATCAAGTGGGCACACCAATCCACACAATCAGGT TGTTTATGACTAATGACTGCGTTAGGACGATTGCCTTGCAATCGGCAAAGGGACAAACATCATCTTCGATCGCAACACCTGCATTGGT GGTCATGGTATCTCAATTGTTTGTCCCTCCCCACCCCTTCTCGTACTGGAAACTTGATCTGCATTATAGGGCTCTATTGGCTCCAATG TCGCTGTTTCTGGCGTCATCGTCACGGGCAACATCATCACCGACAGGTGCGAATTCGGTCACCTCTTTGAAGTTGGTTTGTCAGACTC AATCAAATGCAGTGACCAGGCTCTTCGTATTAAAACTATTGCTTCAGCAACTGGGAGTGTCGTTGGCAACGTCACATACTCTGTAACT AGCACTAACCGTGCATCTTAAACTCGTGGCTGACGAATTTATTACAAGGGAAACACTGCGAAAGGAATCCGAAGATTTGGCGTGATCA TCGACCAGGTACCTCTCTGAATTCCTCACTAATAATTCCTCACTAATACGCCCAATAATAGAGTTATCCCAGCACCCTTGGTACTCCT GGAATGGGCACTATTATTTCGGTATTATATGAAACTGCCTTCTTTGACTGGAGTTGACAAAGGGTTAGAACGTCAACTTTGGTAGCCC CACTTCCAACATCGCCGTGAACAGCGGGGCAAAGCAGGTTGCGGTAAATTGTGGATACGGTTCTTGCACAGGTATGACAGCCATTTTT TTCTGAACATTTCATATGTGATGGTCTGACGCTGTATTAGGTACATGGGACTGGCACTACCTGTCTGTCAAAGGCGGCGTCACAAGTC AGTACATTGTTGATTGAAGAGGGGCCACCAATTCACTTTCATATTAGGTCCTCCCAGCAACGCGAACATACTGGGGCTCTAGAGCCCT TGATGGCAGGCTGACTATCTCCTTAACGTCAAAAATGCCAATGGTCGCTACCACCGGATTCAGGACAAAGAGCCACTCATCACGGATA TGAATCCGAATGGACATTTGATGCCCTCTGCTTTCCTTTTCTTCAGTACTTACCTTCATTTCTTCTTGCACTCTTTTCATCAACGTTC CTTACAACGCACATTACATACAATTTTTT

\section{(d)}

ATGCTCTCTGTTTTCTCCGTATTGCTTTTCGCTGGTGTAGTGTTAGCATGCCAGGGGTCCATAT CGTCTTTGTCTGACGTGGATTTAGCTGTCAAGTGCACCACAATTACACTTCGGGGCTTCACTGT TCCTGCTGGCCAGGGTTTAATTCTGAATCTTTTGCCAGGAACGACGGTTACGATGGCTGGTGAT АTTTCATTTGGCAACAAAAGCTGGGCAGGCCCCCTTTTCACCATAATTGGAACTTCTATCACAT TCAACGGGAACGGCCATAATTTCAATGGTGGAGGTCCCTTCTATTGGGATGGTACGGGTCTCAG 
TTCCGGCACACAAAAGCCAGATCCTATGATGAGGATCAGAATGTCAGGAACTTTTCAGAATGTG ACAGTGGTCAACTCGCCCGCGCGGGTTTTCTCTGTGTCGAATCCCGCACCCTTGCTGATGACAA AGTTGACAGTCGATAACTCTCAAGGAGACTACCCCAATAACAGGAGCTATGGGACTGCCGCTGG CCACAACACCGATGGATTCGATGTGAGCGGCCATGACGTAACGATTCAGAACTGTACGGTTCAT AATCAAGACGATTGCCTTGCAATCGGCAAAGGGACAAACATCATCTTCGATCGCAACACCTGCA TTGGTGGTCATGGTATCTCAATTGGCTCTATTGGCTCCAATGTCGCTGTTTCTGGCGTCATCGT TACGGGCAACATCATCACCGACAGTGACCAGGCTCTTCGTATTAAAACTATTGCTTCAGCAACT GGGAGTGTCGTTGGCAACGTCACATACTCTGGAAACACTGCGAAAGGAATCCGAAGATTTGGCG TGATCATCGACCAGAGTTATCCCAGCACCCTTGGTACTCCTGGAACGGGCACTATTATTTCGAA CGTCAACTTTGGTAGCCCCACTTCCAACATCGCCGTGAACAGCGGGGCAAAGCAGGTTGCGGTA AATTGTGGATACGGTTCTTGCACAGGTACATGGGACTGGCACTACCTGTCTGTCAAAGGCGGCG TCACAAGTCCTCCCAGCAACGCGAACATACTGGGGCTCTAG

\section{(e)}

CATTTTGGTTCAGAAACCACTGGCCATTCCTTCCGACCTGGCGGCACTACTTTCTATGCATTAA ATGGCACCCCCTCCAATGTCATTCAAGGAATGGGTCGTTGTAAATCAGAGGCCTTCCAGTCTTA CATTCATGTACACCССTCCCTGCTGGCCACATTTATTTTCTCTTCAAACAACAGTCCGCTTTGT

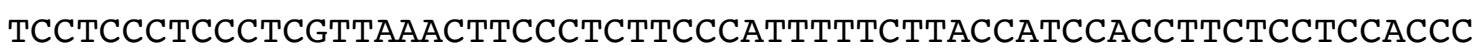
TTTCTTTTCCAAGCTCAGTTTTCTACACTTTTTCTCCATAGTATCTTATGTACCCAGAAAAATG AGACTTGTTTAGCAGGCCCTGCCGCTGGTCACAGGGCAGTGGCCATGCAACCCTATCCCTCGGA ATGACTGCCTGAGCAAAACCTTAAGGGATTAGTCAAGCACTACATGTGATGGTGTGAATCGCTT AGTAACATTAAGCTGAATTTGTCATCCATTGTGTTTTCATGTATAGACATTGTGTTCACCCCTC AGATGACTCAACCACAGCGCAACAAATGGGTAGGAGGCTGCCATATCTCCAACCTCATCCTTGT ACTTTACATGCAACCTCCTTGGAGGATCTTCCTGCCCACСTTCTCCACAAGATCTTCATATTCT GTTGCCTGCGACACCTGTAGACTGACTTCACCCACTCCCTCTCTTATTATCGTTCAAATGGTTT GAACGATCGTGGGTGCTTCAATGGGAATTATAGGATGGCATCGGTGGTGGCACAACCATCTTCA GCACATATGCAGTTGGTTGACTTGCACTCTGGGATCGGTTCATGAGAGGCGCCGTCGACCTGAT CTTATGTAAGATATTGGAGGGTTGACATTTTTGGTTCAGGGCTTGCTCAGGCATTGTTGTGGCA ATGTCCGTGCCACACATGGTCAAATATTAGCAAACACATCTGGATCTGTCTCTGAGTCTTTCAA GTTGAATCCCTCTCCATGGAGCGAAAATCCAGAAGGCTGAGTACTTATTGAAACCTGAAACGGC GAAAAACAATACTATCTTCAAGCAGCCCTTGATCTTGGACAGCGGATAACTCGCACTGCACCCT GTCTCTGTCTAATGGAGAAAAAAAATTCCACTGCTGTCATGGTGCATAAGGGGACTGGTTTCAA TTTGATCAAGAAAGAAATTCGACGACTCAATTGCAATTCTCTTGGCTTTATGCTGGTCTTCTCT ATTTGACTTGATCTCAACGCTTTGAGGCTGCCACGATGGGAGAAGGCTTCAGAAGTTCGCAATG CCAGAACTTTGAACCCTCTCAGTCTCCACAGCCGTTATCCTTCCTGCGTAGCTACTAGGGTCGA GGGACTCCAGGAACCTCACGGTCGATCTGGGTCACGTTTATGAGAGGTCGAAGAGGCGGTTCTC GCAATCGCAGATTTAAGCTCTAGATTAAAAGCGACGCAACAATCTCGCGGTAACACAAAAGCTA AATGGGGTGGATGCAATGGTCGCAAAGAGAAGACCTGAATGGAGGAACGCCTACTGCTTAGCGC CATTTACAAGGCGCTACTGTAGCAACCCAACTTGTGTTATTGGTTTGAATTCTATGCTTGAAGT GCTCATGACGACAGAGTTTTGATGTCCGGAAGTCATTTATCCGAATTCTCTGCGATTCTTGGAT 
CTGGCGAGATCCCTCCACTAATCTCTTCCTCCGAGCGGCCAATTAACTCAACTTGAGGAGATAT AAATGCTCGAGGGAAACTTTGGCGACATTCAAC

(f)

CACAATTACACTTCGGGGCTTCACTGTTCCTGCTGGCCAGGGTTTAATTCTGAATCTTTTGCCA GGAACGACGGTTACGATGGCTGGTGATATTTCATTTGGCAACAAAAGCTGGGCAGGCCCCCTTT TCACCATAATTGGAACTTCTATCACATTCAACGGGAACGGCCATAATTTCAATGGTGGAGGTCC СTTCTATTGGGATGGTACGGGTCTCAGTTCCGGCACACAAAAGCCAGATCCTATGATGAGGATC AGAATGTCAGGAACTTTTCAGAATGTGACAGTGGTCAACTCGCCCGCGCGGGTTTTCTCTGTGT CGAATCCCGCACCCTTGCTGATGACAAAGTTGACAGTCGATAACTCTCAAGGAGACTACCCCAA TAACAGGAGCTATGGGACTGCCGCTGGCCACAACACCGATGGATTCGATGTGAGCGGCCATGAC GTAACGATTCAGAACTGTACGGTTCATAATCAAGACGATTGCCTTGC 
Figure S2. The L. amethystina GH28A polygalacturonase. (A) Amino acid sequence; (B)

Alignment of $L$. bicolor and L. amethystina GH28A protein sequences.

(A)

MLSVFSVLLFAGAVLVAEACQGSISSLSDVDYAVKCTTITLRGFTVPAGQGLILNLLPGTTVTM

AGDISFGNKSWAGPLFTIIGTSITFNGNGHKFNGGGPFYWDGKGLNSGTQKPDPMMKIKMSGTF

QGVTVVNSPARAFSVANPAPLLMTGLTVDDSQGDYANNRSKGLAAGHNTDGFDVGSDNVTIQNC

TVHNQDDCLAINKGKNITFDRNTCIGGHGISIGSIGSNVAVSGVIVSNNIINESDQALRIKTIA

SATGSVVGNVTYSGNTAKGIRKFGVIIDQSYPSTLGTPGTGTIISNVNFGSPF SNITVNSGANQ

VAVNCGSGSCIGTWNWYYLSAKGGVTSPPSNVNILGL

(B)

\begin{tabular}{|c|c|}
\hline \multirow{3}{*}{$\begin{array}{l}\text { LbGH28_A } \\
\text { LaGH28 }\end{array}$} & MLSVFSVLLFAG---VVLACQGSISSLSDVDLAVKCTTITLRGFTVPAGQGLILNLLPGT \\
\hline & MLSVFSVLLFAGAVLVAEACQGSISSLSDVDYAVKCTTITLRGFTVPAGQGLILNLLPGT \\
\hline & 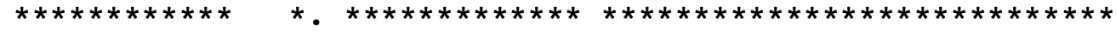 \\
\hline LbGH28_A & TVTMAGDISFGNKSWAGPLFTIIGTS ITFNGNGHNFNGGGPFYWDGTGLSSGTQKPDPMM \\
\hline LaGH28 & 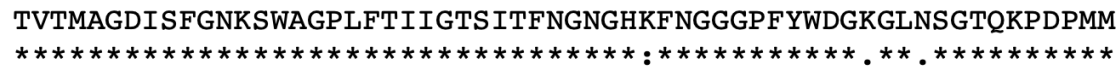 \\
\hline LbGH28_A & RIRMSGTFQNVTVVNSPARVFSVSNPAPLLMTKLTVDNSQGDYPNNRSYGTAAGHNTDGF \\
\hline LaGH28 & $\begin{array}{l}\text { KIKMSGTFQGVTVVNSPARAFSVANPAPLLMTGLTVDDSQGDYANNRSKGLAAGHNTDGF } \\
: *: * * * * * * * * * * * * * * . * * *: * * * * * * * * * * *: * * * * * * * * * * * * * * * * * *\end{array}$ \\
\hline LbGH28_A & DVSGHDVTIQNCTVHNQDDCLAIGKGTNI IFDRNTCIGGHGISIGS IGSNVAVSGVIVTG \\
\hline LaGH 28 & $\begin{array}{l}\text { DVGSDNVTIQNCTVHNQDDCLAINKGKNITFDRNTCIGGHGISIGSIGSNVAVSGVIVSN } \\
* * \ldots: \ldots * * * * * * * * * * * * * . * * . * * * * * * * * * * * * * * * * * * * * * * * * * \text { : }\end{array}$ \\
\hline LbGH28_A & NIITDSDQALRIKTIASATGSVVGNVTYSGNTAKGIRRFGVIIDQSYPSTLGTPGTGTII \\
\hline LaGH 28 & 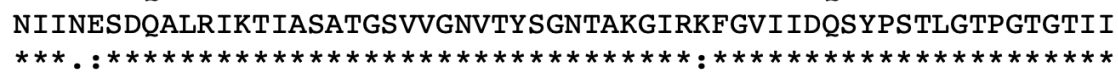 \\
\hline LbGH28_A & SNVNFGSPTSNIAVNSGAKQVAVNCGYGSCTGTWDWHYLSVKGGVTSPPSNANILGLMGN \\
\hline LaGH 28 & $\begin{array}{l}\text { SNVNFGSPFSNITVNSGANQVAVNCGSGSCIGTWNWYYLSAKGGVTSPPSNVNILGL--- } \\
* * * * * * * * * *: * * * * *: * * * * * * * * * * * *: *: * * * . * * * * * * * * * . * * * * *\end{array}$ \\
\hline LbGH28_A & MLSV \\
\hline LaGH2 8 & ---- \\
\hline
\end{tabular}




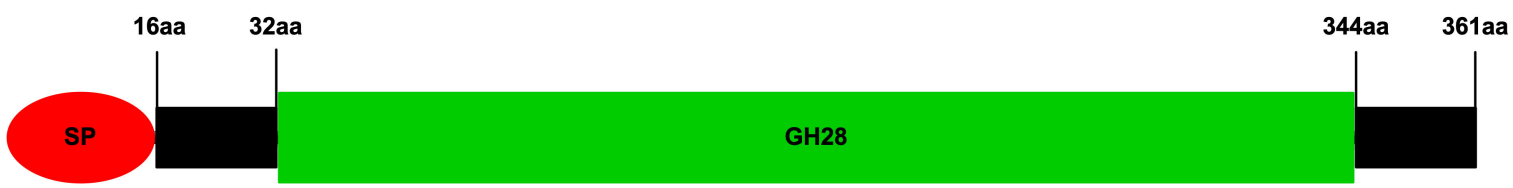

b

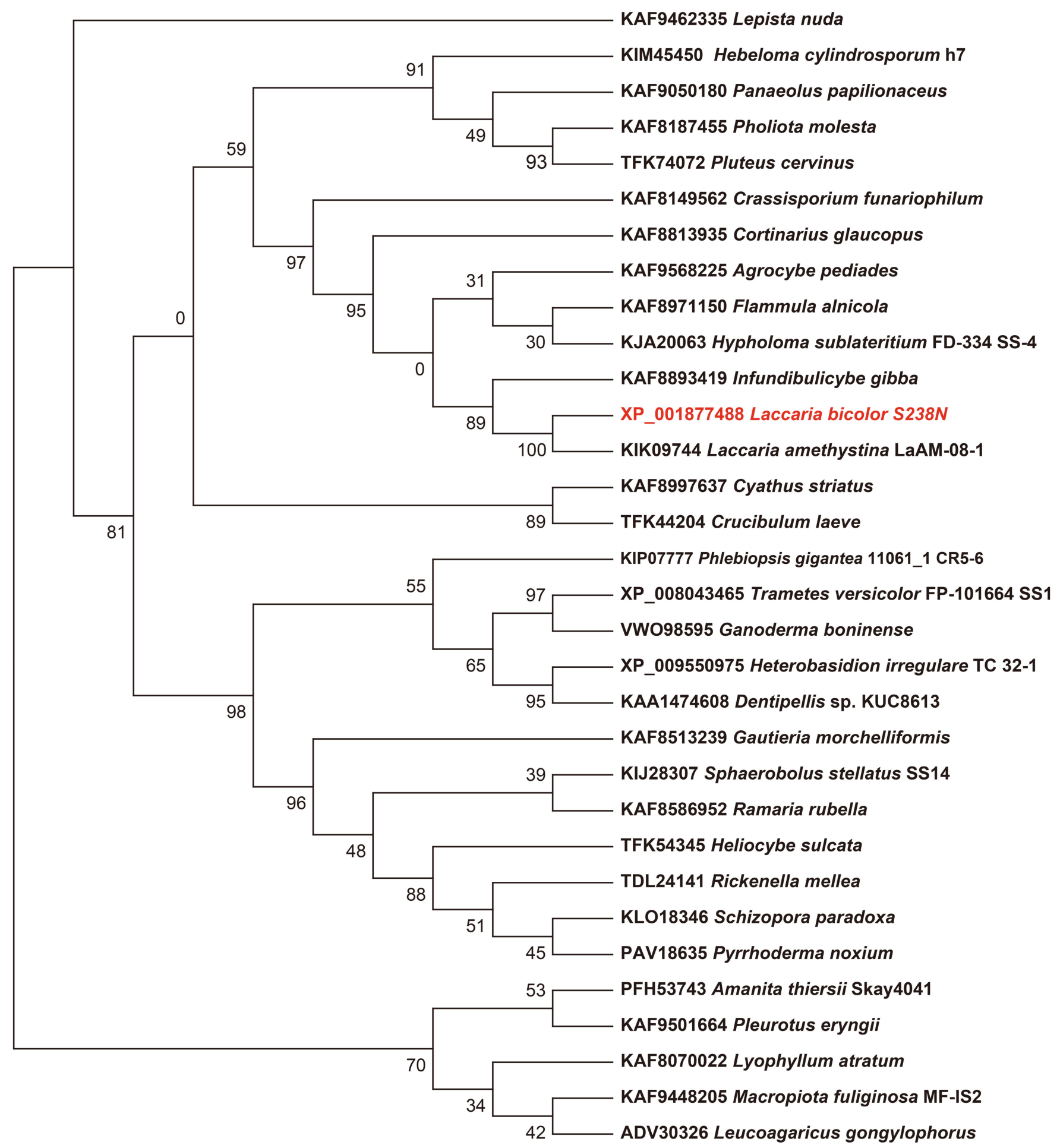


Fig. 3

bioRxiv preprint doi: https://doi.org/10.1101/2021.09.24.461608; this version posted September 24, 2021. The copyright holder for this preprint (which was not certified by peer review) is the author/funder, who has granted bioRxiv a license to display the preprint in

a

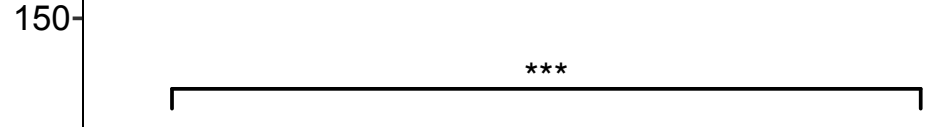

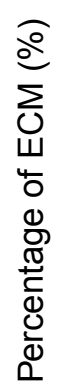

c

0

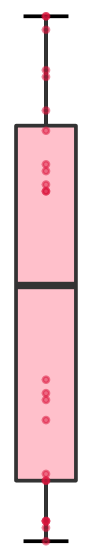
perpetuity. It is made available und

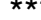
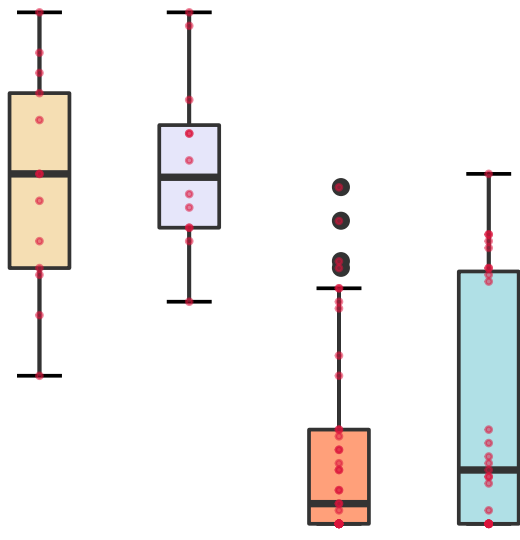

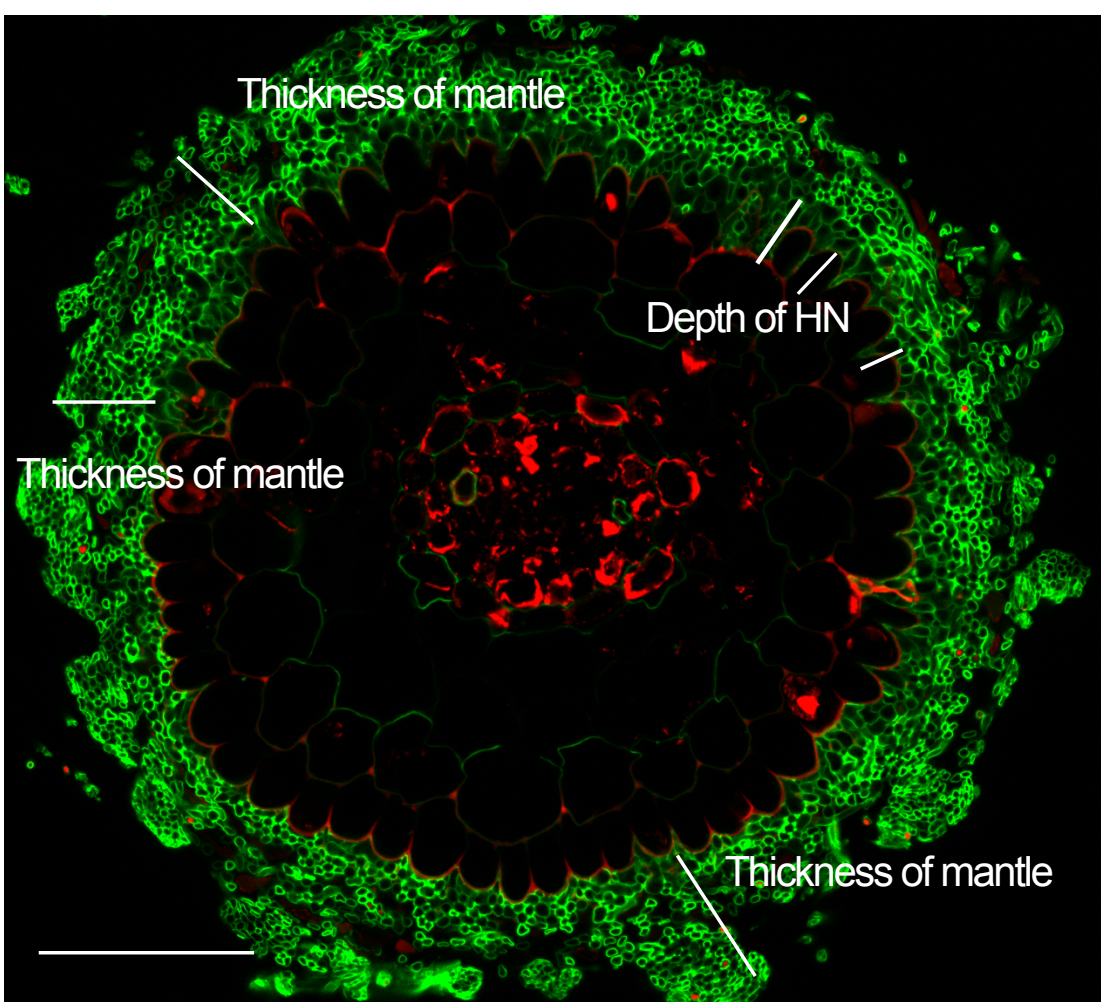

d

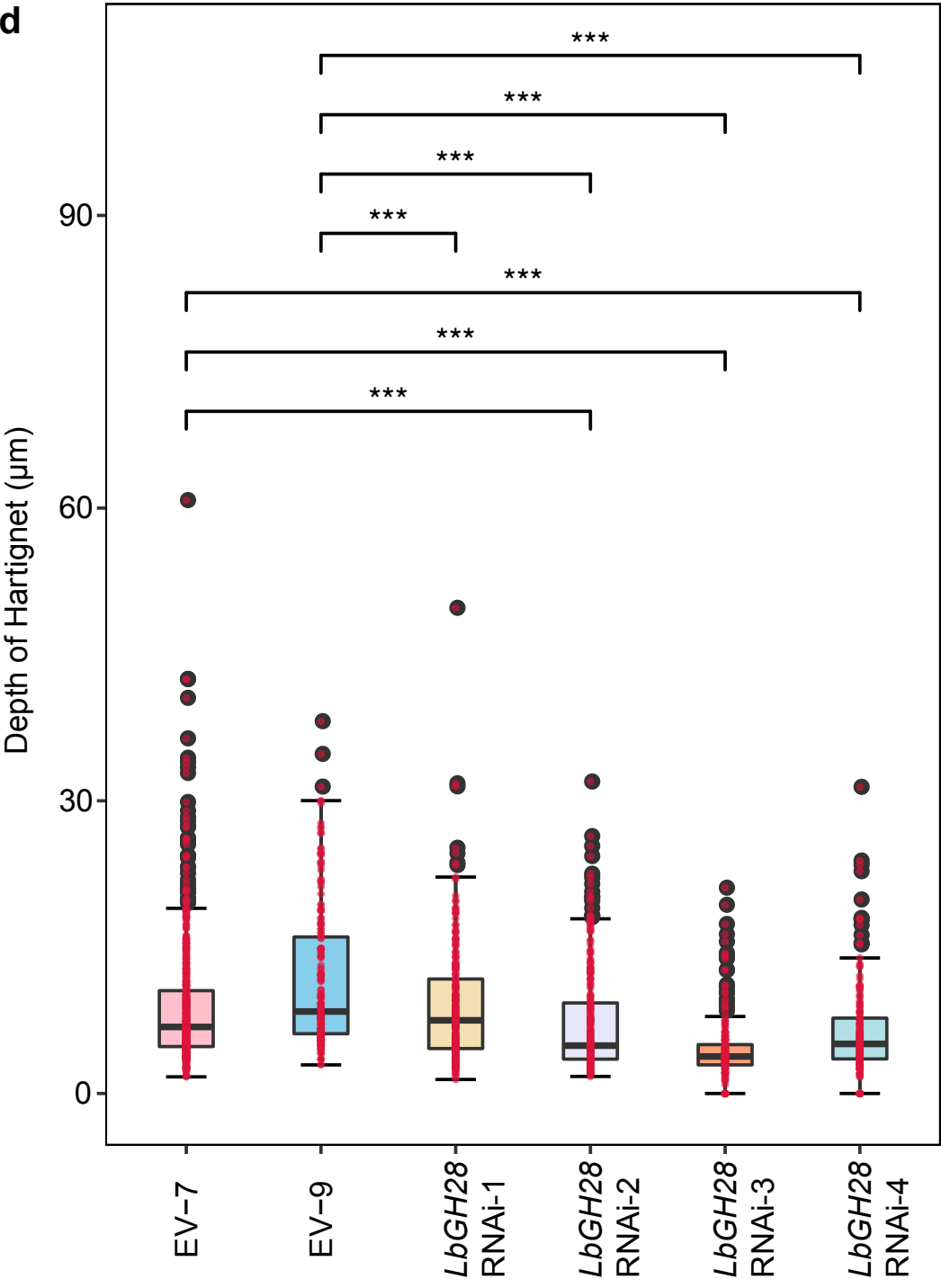


Fig. 4
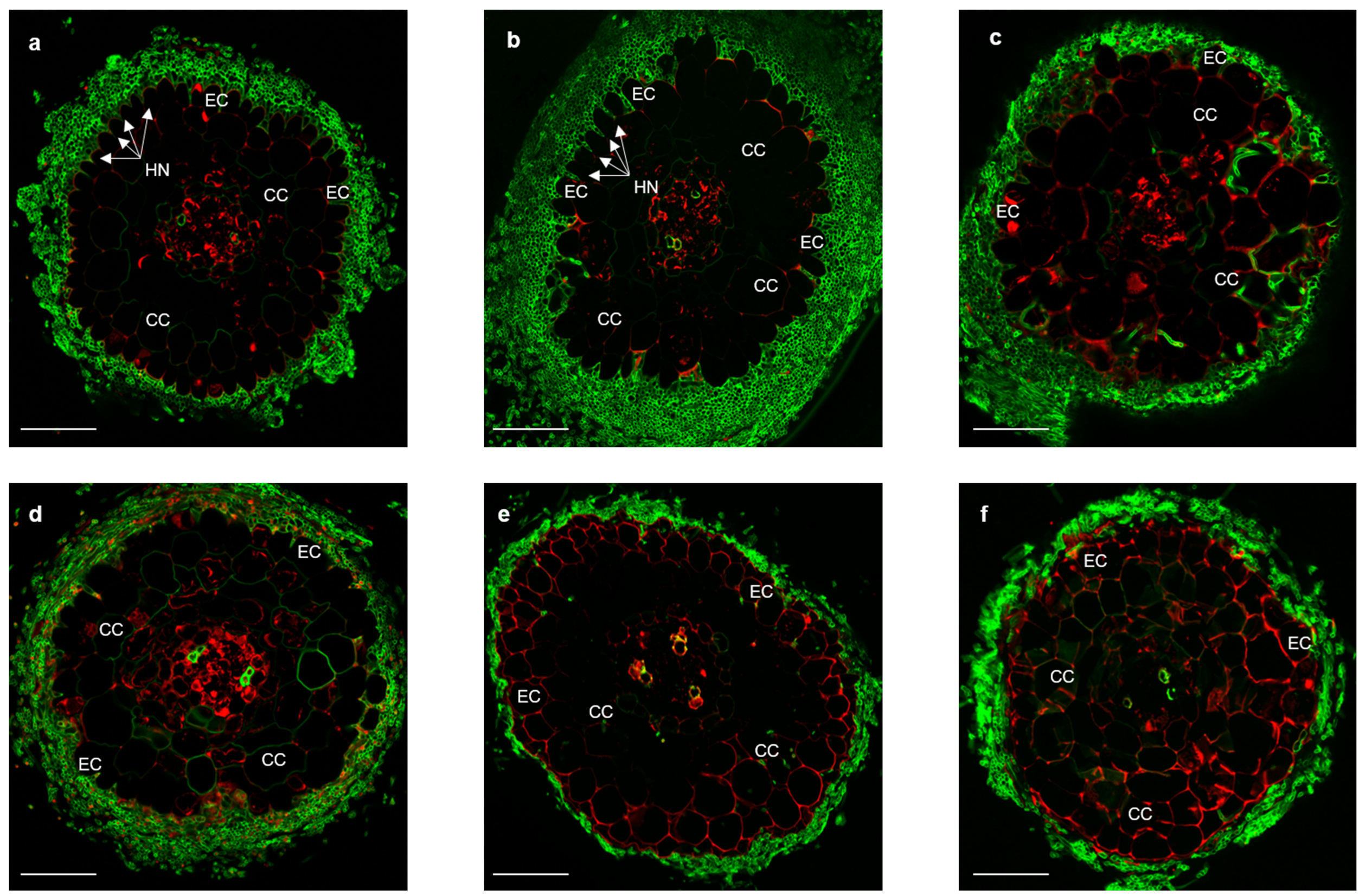
Fig. 5
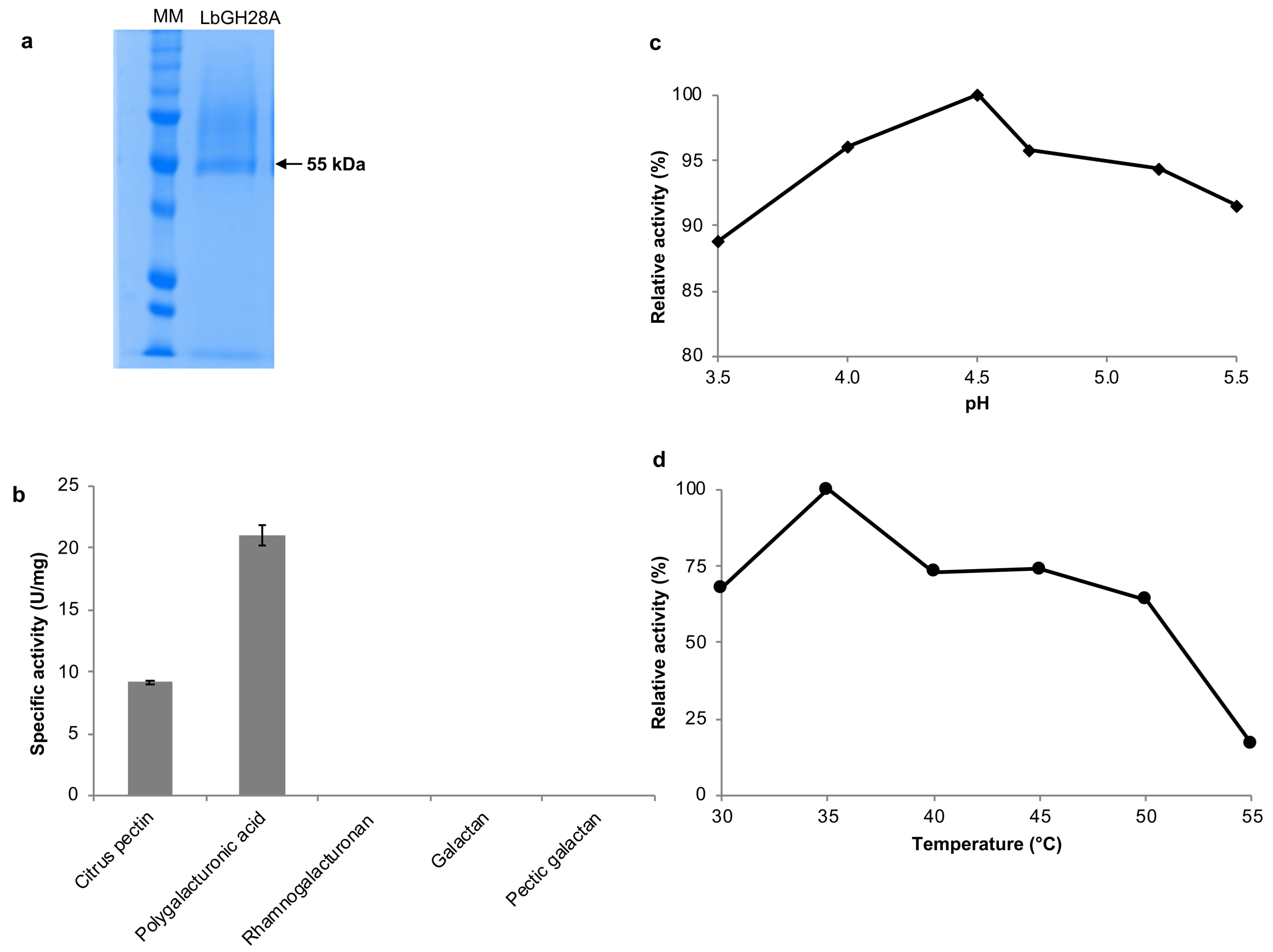
a bo गुराइ cc

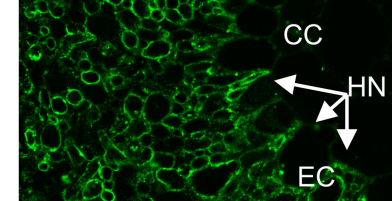

CS EC

: 80 o $\mathrm{M}$

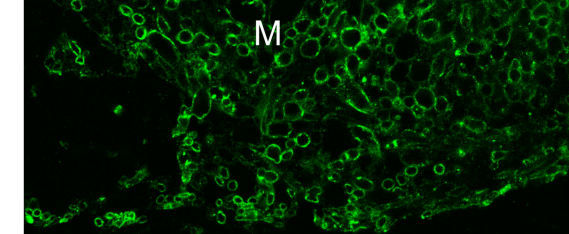

$-$

b

$\overbrace{E C}^{\mathrm{CC}}$

M

M

$\mathrm{T}_{\mathrm{CC}}^{\mathrm{HN}}$

M

$\underbrace{\mathrm{CC}} \mathrm{HN}$ 
bioRxiv preprint doi: $\mathrm{https}$ //doi org/101101/2021.09,24 461608; this version posted September 24, 2021. The copyright holder for this preprint (which was not certified by peer review) is the author/funder, who has granted bioRxiv a license to display the preprint in perpetuity. It is made available under aCC-BY-NC-ND 4.0 International license.

Fig. 7

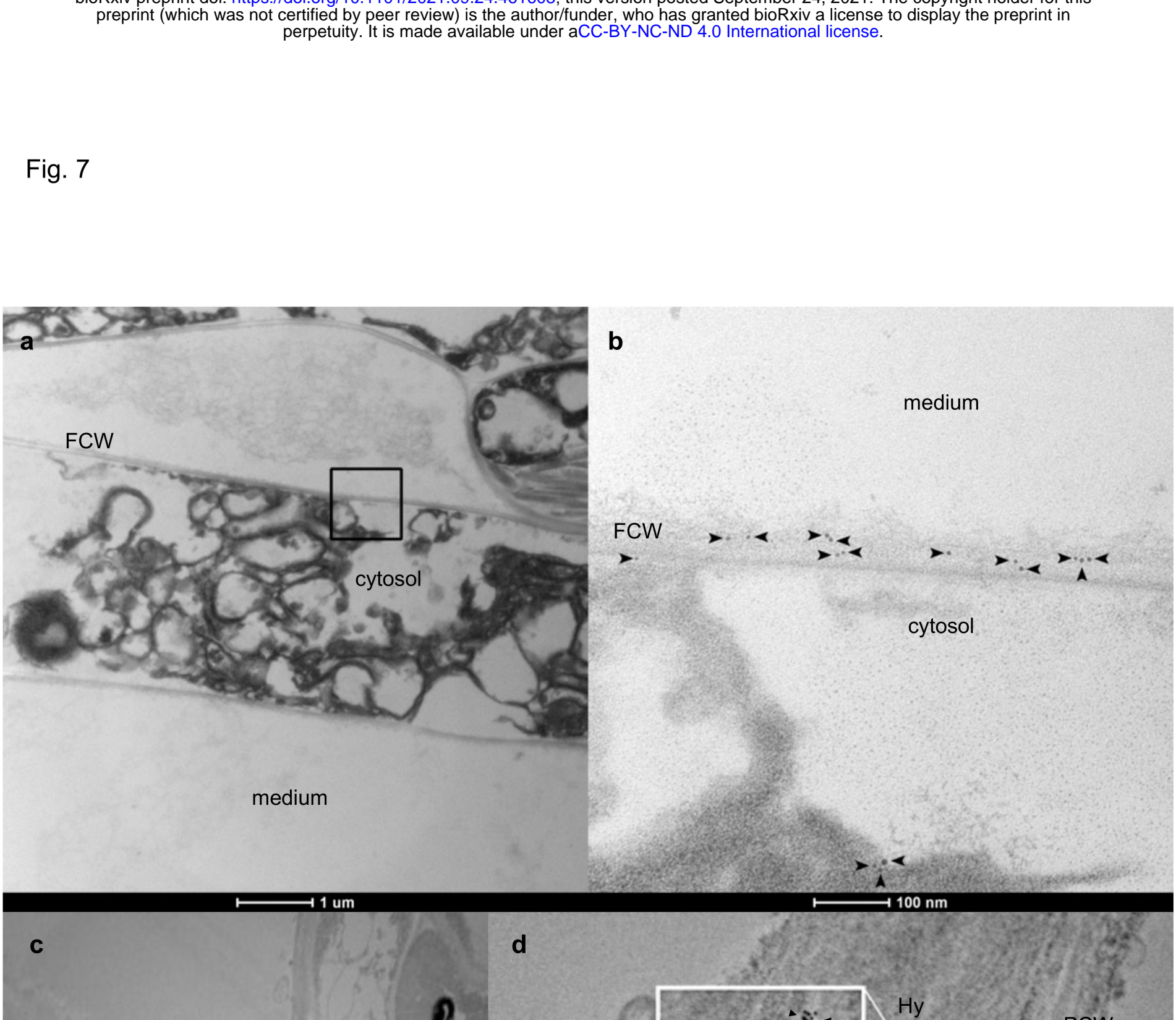

c

d

Hy
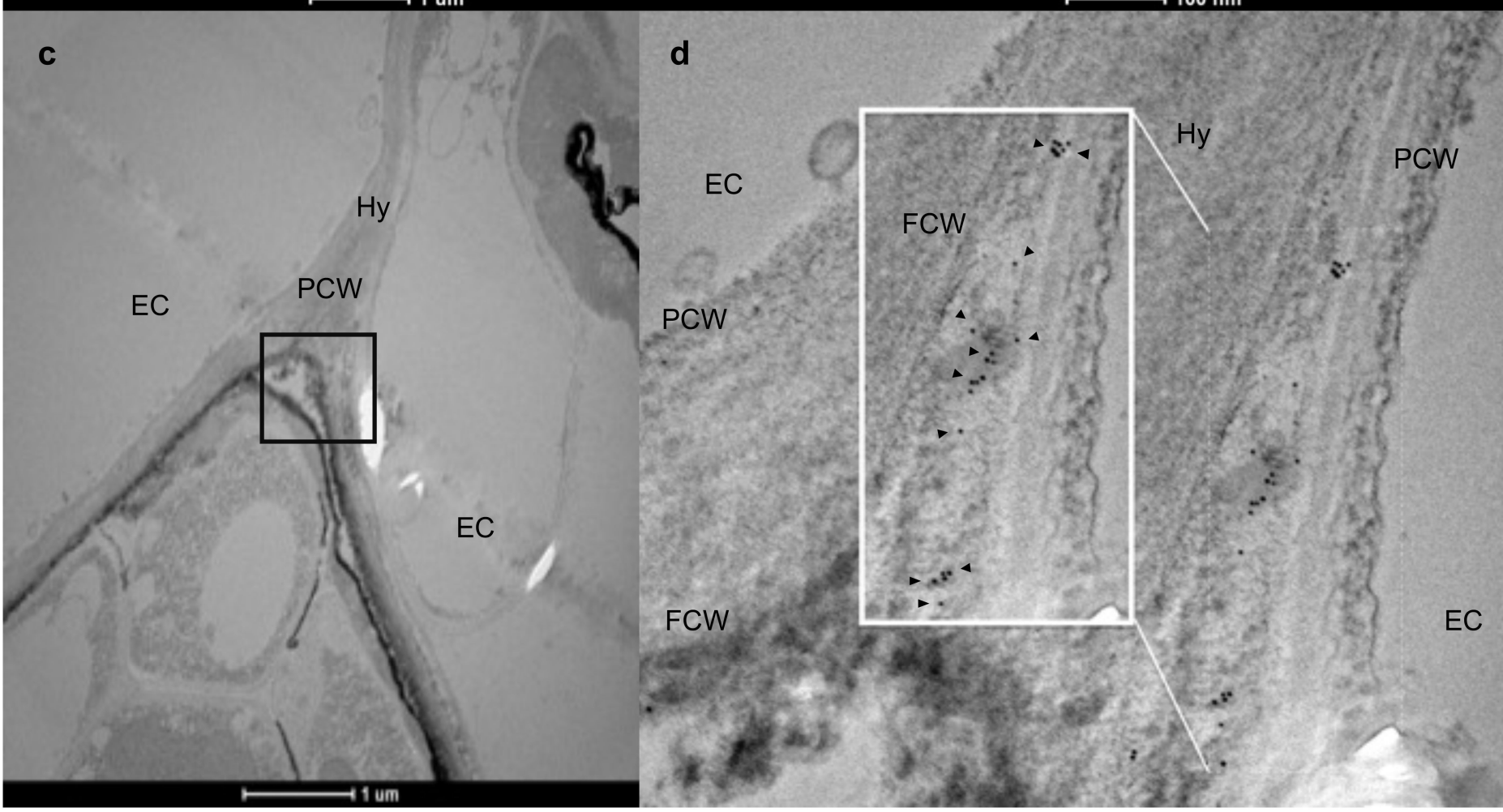

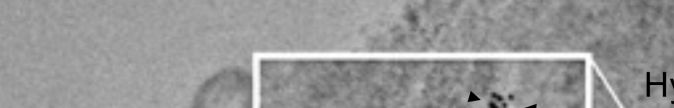

EC

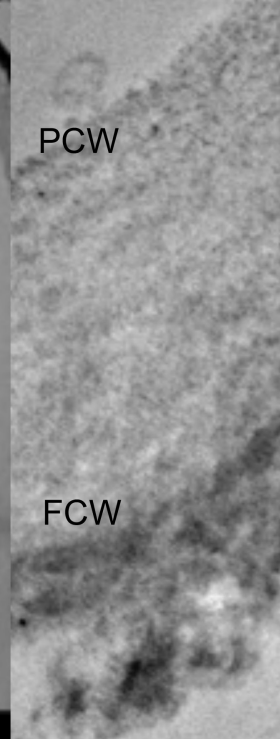

3.8.9.

לy:

PCW

EC 
bioRxiv preprint doi: https://doi org/101101/2021.09.24 461608; this version posted September 24, 2021. The copyright holder for this

preprint (which was not certified by peer review) is the author/funder, who has granted bioRxiv a license to display the preprint in perpetuity. It is made available under aCC-BY-NC-ND 4.0 International license.

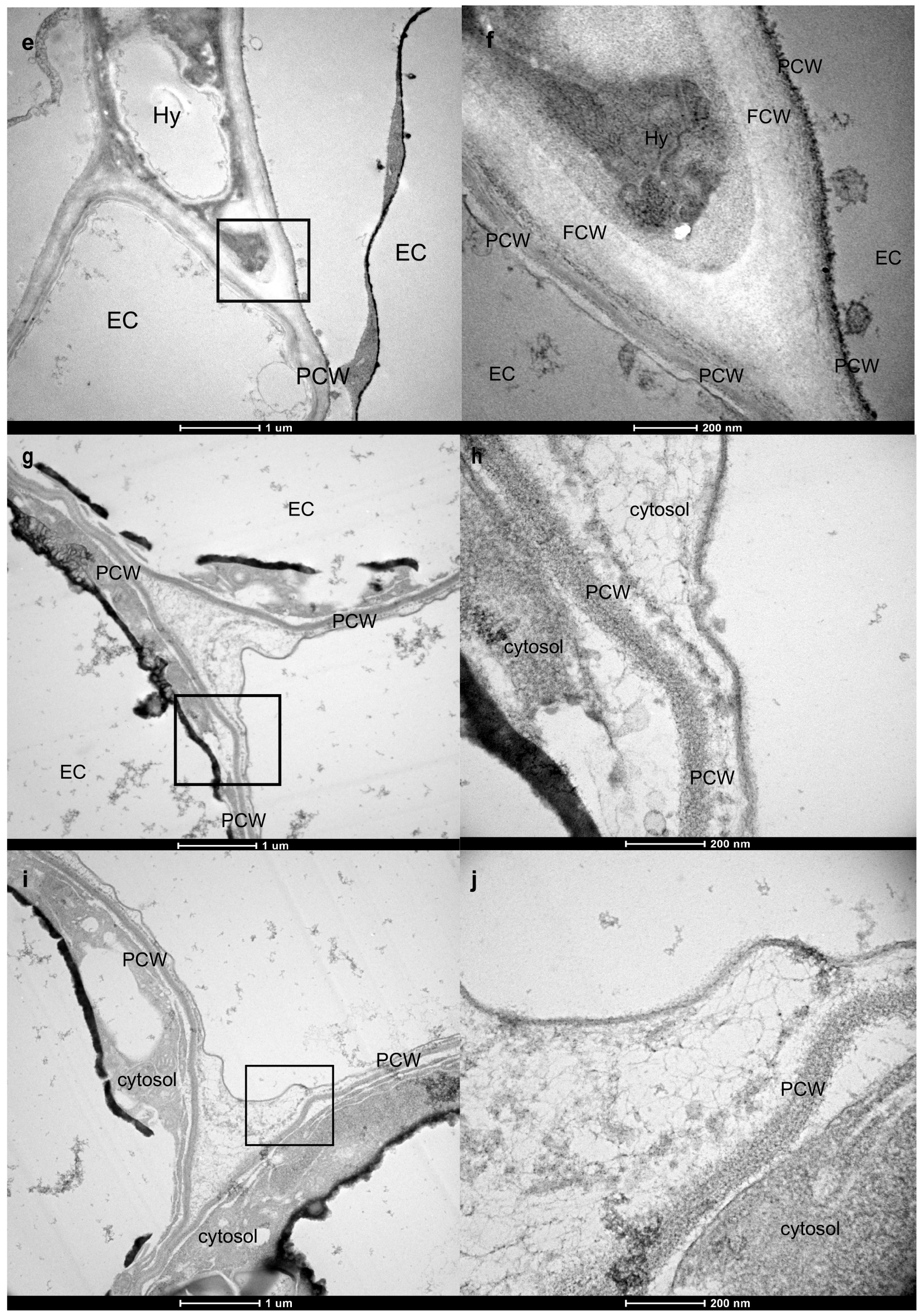


bioRxiv preprint doi: https://doi org/10.1101/2021.09.24 461608; this version posted September 24,2021. The copyright holder for this preprint (which was not certified by peer review) is the author/funder, who has granted bioRxiv a license to display the preprint in perpetuity. It is made available under aCC-BY-NC-ND 4.0 International license.

Fig. 8

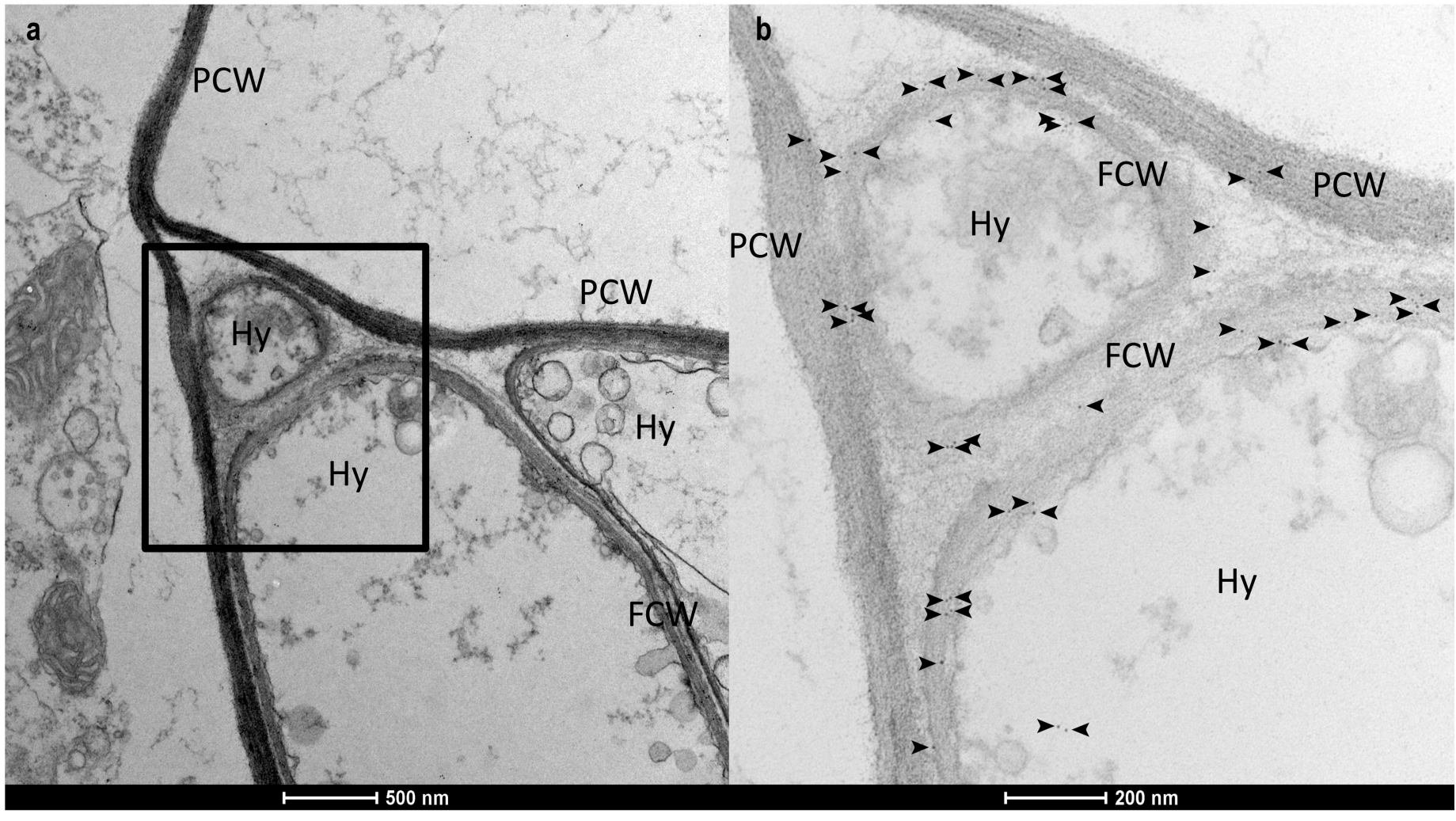

